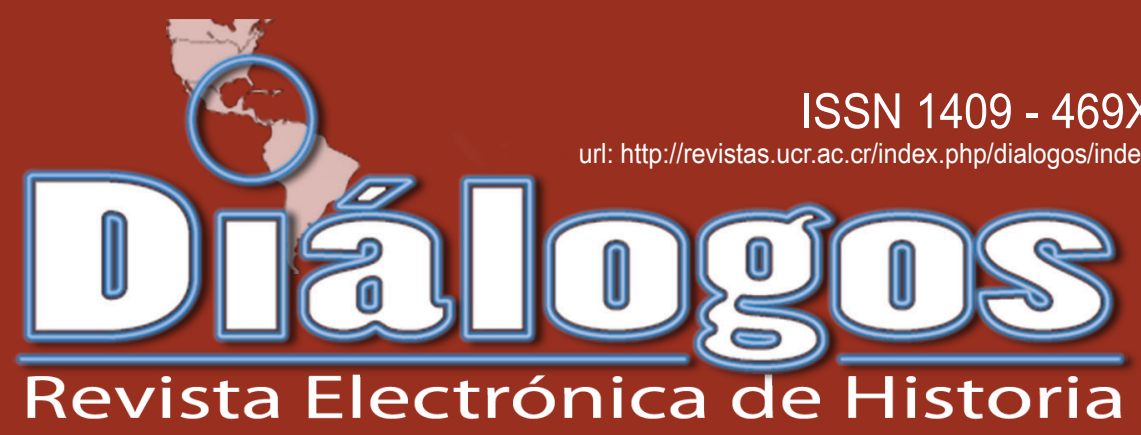

Escuela de Historia. Universidad de Costa Rica Volumen 14 especial en homenaje a Victor Hugo Acuña. Octubre 2013

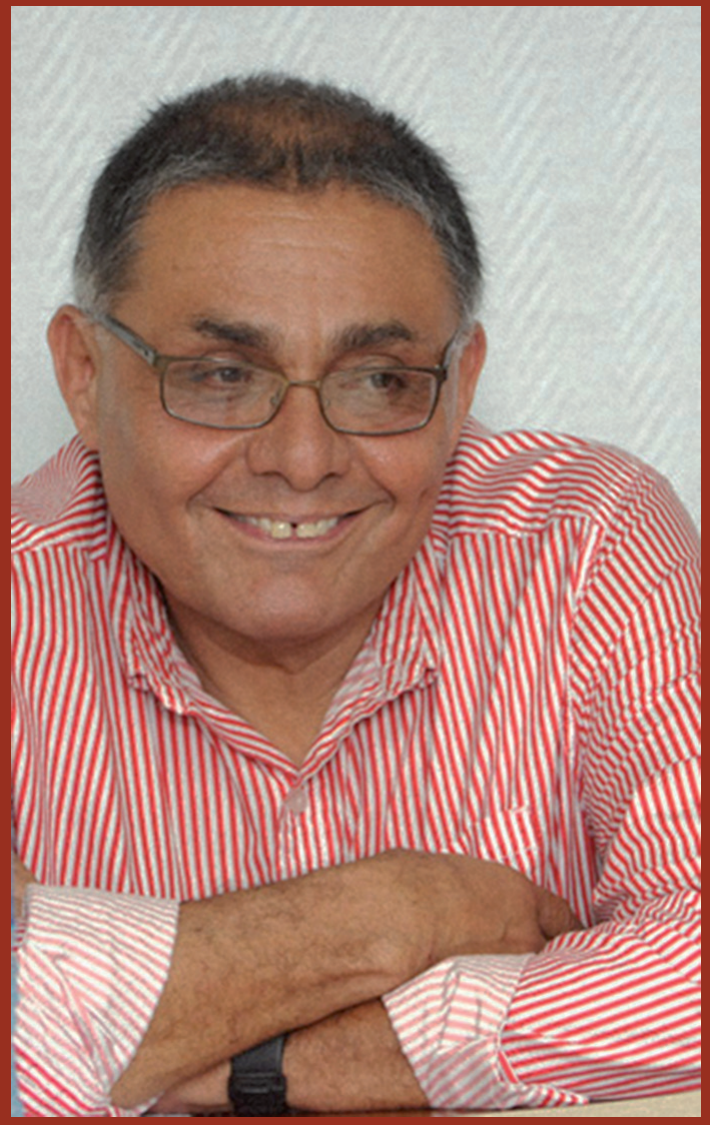

Director de la Revista: Dr. Juan José Marín Hernández juan.marinhernandez@ucr.ac.cr

Editor académico: Dr. Ronny Viales Hurtado - ronny.viales@ucr.ac.cr Editor técnico: M.Sc. Marcela Quirós G. - marcela.quiros@ucr.ac.cr 


\section{Miembros del Consejo Editorial:}

Dr. Juan José Marín Hernández, Catedrático. Director del Centro de Investigaciones Históricas de América Central. Universidad de Costa Rica. Costa Rica. juan. marin@ucr.ac.cr

Dr. Ronny Viales Hurtado. Catedrático. Historia Económica y Social. Universidad de Costa Rica. Director de la Escuela de Historia. Costa Rica. ronny. viales@ucr.ac.cr

Dr. David Díaz Arias: Catedrático. Historia Política, Director del posgrado de Historia y Docente de la Escuela de Historia, Universidad de Costa Rica, Costa Rica.david.diaz@ucr.ac.cr

MSc. Francisco Enríquez. Historia Social. Universidad de Costa Rica. Costa Rica. francisco.enriquez@ucr. ac.cr

Dra. Ana María Botey. Historia de los movimientos sociales. Universidad de Costa Rica. Costa Rica. abotey@gmail.com

\section{Miembros del Consejo Asesor Internacional:}

Dr. José Cal Montoya. Universidad de San Carlos de Guatemala.Guatemala. jecalm@correo.url.edu.gt

Dr. Juan Manuel Palacio. Universidad Nacional de San Martín. Argentina.jpalacio@unsam.edu.ar

Dr. Eduardo Rey. Universidad de Santiago de Compostela. España. ereyt@usc.es

Dr. Heriberto Cairo Carou. Departamento de Ciencia Política y de la Administración III Universidad Complutense de Madrid. España. hcairoca@cps.ucm.es

Dra. Rosa de la Fuente. Departamento de Ciencia Política y de la Administración III Universidad Complutense de Madrid. España. rdelafuente@cps. ucm.es

Dr. Javier Franzé. Departamento de Ciencia Política y de la Administración III Universidad Complutense de Madrid. España. javier.franze@cps.ucm.es

Dr. Jaime Preciado Coronado Departamento de Estudios Ibéricos y Latinoamericanos. Universidad de Guadalajara. México. japreco@hotmail.com
Dr. Gerónimo de Sierra. Vicerrector de la Universidade Federal da Integração Latino-Americana (UNILA) y Departamento de Sociología, Facultad de Ciencias Sociales de la Universidad de la República. Uruguay. geronimo@fcs.edu.uy

Dr. Antonio Palazuelos. Departamento de Ciencia Política y de la Administración III - Universidad Complutense de Madrid. España. palazuelosa@cps. ucm.es

Dr. Werner Mackenbach. Universidad Potsdam. Alemania. werner.mackenbach@uni-potsdam.de

Dr. Guillermo Castro. Ciudad del Saber Panamá. Panamá.gcastro@cdspanama.org

Dra. Natalia Milanesio. University of Houston. Estados Unidos.nmilane2@Central.UH.EDU

Dr. Ricardo González Leandri. Consejo Superior de Investigaciones Científicas - España. España. rgleandri@gmail.com

Dra. Mayra Espina. Centro de Estudios Psicológicos y Sociológicos, La Habana. Cuba.mjdcips@ceniai.inf.cu

Dra. Montserrat Llonch. Departamento de Economía e Historia Económica Universidad Autónoma de Barcelona. España.montserrat.llonch@uab.es

Dra. Estela Grassi. Universidad de Buenos Aires. Argentina. estelagrassi@gmail.com

Dra. Yolanda Blasco. Universidad de Barcelona. España. yolandablasco@ub.edu

Dr. Alfredo Falero. Departamento de Sociología. Universidad de la República. Uruguay. alfredof@adinet. com.uy

\section{Portada:}

Fotografía de Anel Kenjekeeva, Oficina de Divulgación Universidad de Costa Rica, publicada en http://www. ucr.ac.cr/noticias/2012/09/13/escuela-de-historia-rindehomenaje-a-victor-hugo-acuna.html

\section{Equipo Técnico Editorial:}

$\begin{array}{ll}\text { Editora Técnica: } & \text { M.Sc. Marcela Quirós Garita. } \\ & \text { marcela.quiros@ucr.ac.cr } \\ \text { Diagramación: } & \text { Cindy Chaves Uribe } \\ \text { Asistentes: } & \begin{array}{l}\text { Pablo Hurtado Granados } \\ \text { Maureen Méndez Montero }\end{array}\end{array}$


"Diálogos Revista Electrónica de Historia" se publica desde octubre de 1999.

\section{Diálogos está en los siguientes repositorios:}

Dialnet

http://dialnet.unirioja.es/servlet/

revista?tipo_busqueda=CODIGO\&clave_revista $=3325$

Latindex

http://www.latindex.unam.mx/larga.php?opcion=1\&folio=12995;

\section{UCRindex}

http://www.revistas.ucr.ac.cr/

\section{Scielo}

http://www.scielo.cl/

\section{eRevistas}

http://www.erevistas.csic.es/

\section{REDALYC}

http://redalyc.uaemex.mx/src/inicio/FrmBusRevs2.jsp?iEdoRev=2\&cvepai=11;

\section{LANIC}

http://lanic.utexas.edu/la/ca/cr/indexesp.html

Repositorio de Revistas Universidad de Costa Rica http://www.latindex.ucr.ac.cr/

Directorio y recolector de recursos digitales del

Ministerio de Cultura de España

http://roai.mcu.es/es/inicio/inicio.cmd

DOAJ Directory of open access \& Hybrid journals

http://www.doaj.org/doaj?func=byTitle\&hybrid=1\&query=D

Biblioteca de Georgetown

http://library.georgetown.edu/newjour/d/msg02735.html

Asociación para el Fomento de los Estudios Históricos

en Centroamérica

http://afehc. apinc.org/index.php?action=fi_aff\&id=1774

Universidad de Saskatchewan, Canadá

https://library.usask.ca/ejournals/view/1000000000397982

\section{Monografias}

http://www.monografias.com/Links/Historia/more12.shtml

\section{Hispanianova}

http://hispanianova.rediris.es/general/enlaces/hn0708.htm

Universidad del Norte, Colombia

http://www.uninorte.edu.co/publicaciones/memorias/enlaces.htm

Universidad Autónoma de Barcelona

http://seneca.uab.es/historia/hn0708.htm

Repositorio Invenia - Gestión del Conocimiento

http://www.invenia.es/oai:dialnet.unirioja.es:ART0000086144

\section{Enlace Académico}

http://www.enlaceacademico.org/biblioteca/

revistas-en-formato-digital-centroamerica/

\section{Electronic Resources}

http://sunzi1.lib.hku.hk/ER/detail/hkul/3987318

Revistas académicas en texto completo

http://web.prw.net/ vtorres/
Diálogos se anuncia en las siguientes

instituciones y sitios académicos:

Maestroteca

http://www.maestroteca.com/detail/553/dialogos-revista-electronica-de-historia.html

Biblioteca de Georgetown

http://library.georgetown.edu/newjour/d/msg02735.html

Asociación para el Fomento de los Estudios Históricos en Centroamérica

http://afehc. apinc.org/index.php?action=fi_aff\&id=1774

Universidad de Saskatchewan, Canadá

https://library.usask.ca/ejournals/view/1000000000397982

\section{Monografias}

http://www.monografias.com/Links/Historia/more12.shtml

\section{Hispanianova}

http://hispanianova.rediris.es/general/enlaces/hn0708.htm

Universidad del Norte, Colombia

http://www.uninorte.edu.co/publicaciones/memorias/enlaces.htm |

Universidad Autónoma de Barcelona

http://seneca.uab.es/historia/hn0708.htm

Repositorio Invenia - Gestión del Conocimiento

http://www.invenia.es/oai:dialnet.unirioja.es:ART0000086144

\section{Enlace Académico}

http://www.enlaceacademico.org/biblioteca

revistas-en-formato-digital-centroamerica/

\section{Electronic Resources}

http://sunzi1.lib.hku.hk/ER/detail/hkul/3987318

Revistas académicas en texto completo

http://web.prw.net/ vtorres/

La revista electrónica Diálogos es financiada por

Vicerrectoría de Investigación de la Universidad de Costa Rica
Dialnet - eRevistas

- UCRindex - Latindex -

REDALYC - DOJAC - Directorio

y recolector de recursos

digitales del Ministerio de

Cultura de España

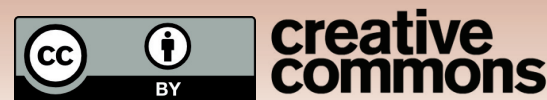




\title{
BIOBIBLIOGRAFÍA DEL DR. VÍCTOR HUGO ACUÑA ORTEGA, HISTORIADOR COSTARRICENSE
}

\section{BIO-BIBLIOGRAPHY OF DR. VÍCTOR HUGO ACUÑA ORTEGA-COSTA RICAN HISTORIAN}

Vanessa Alvarado-Barrientos

\author{
Palabras clave \\ Biobibliografía, Costa Rica, Historiografía.
}

\section{Keywords}

Bio-bibligraphy, Costa Rica, Historiography

Fecha de recepción: 17 de mayo, 2013 - Fecha de aceptación: 8 de agosto, 2013

\begin{abstract}
Resumen
Este trabajo constituye una biobibliografía sobre el historiador costarricense Víctor Hugo Acuña Ortega. Esta biobibliografía tiene como objetivo rescatar la labor que ha realizado Acuña Ortega en su vida como historiador y servir de fuente de información secundaria para futuras investigaciones.
\end{abstract}

\begin{abstract}
This essay introduces a bio bibliography of Costa Rican historian Víctor Hugo Acuña Ortega. Its objetive is to rescue Acuña Ortega's work as a historian in order to offer an important seconday source for otther researchers.
\end{abstract}




\section{BIOBIBLIOGRAFÍA DEL DR. VÍCTOR HUGO ACUÑA ORTEGA, HISTORIADOR COSTARRICENSE}

Vanessa Alvarado-Barrientos

\section{INTRODUCCIÓN}

La elaboración de bibliografías es vista por algunos autores como una ciencia, por otros como una técnica o también como un arte. ${ }^{1}$ Sin embargo, no importa como sea vista, la confección de estas mantienen su validez y utilidad a través de los años. Una bibliografía consiste en listas de documentos donde estos se describen y se clasifican, con el fin último que pueda servir como un instrumento de investigación. Es decir, que sus objetivos principales son identificar a los documentos e informar de su contenido y localización.

Dentro de las bibliografías existen diferentes tipos, una de ellas es la biobibliografía individual que recoge los escritos de un autor en particular y además incluye datos biográficos sobre el mismo. ${ }^{2}$

La presente investigación consiste en una biobibliografía sobre el historiador costarricense Víctor Hugo Acuña Ortega. Responde a uno de los requisitos del curso de Bibliografía Nacional y Latinoamericana del la Carrera de Bibliotecología y Ciencias de la Información de la Universidad de Costa Rica, impartido por la profesora Sonia Pineda Lima. Dicho curso, además de introducir a los estudiantes en las técnicas para realizar este tipo de investigaciones; tiene como propósito rescatar la vida y obra de grandes hombres y mujeres costarricenses que han dejado huella en los distintos campos del quehacer humano.

El Doctor en Historia, Víctor Hugo Acuña Ortega, es una persona que ha servido a la Universidad de Costa Rica por casi 30 años de su vida. Durante este periodo, ha desempeñado cargos de docente a nivel de grado como de posgrado, Director y Subdirector del Centro de Investigaciones Históricas de América Central (CIHAC), presidente de la Asociación Pro Historia Centroamericana, entre otros. Su aporte no ha sido sólo en el ámbito nacional, sino también a escala internacional; ha estudiado, investigado, participado en simposios y conferencias e impartido lecciones en varios países de Centroamérica y Europa.

Esta biobibliografía tiene como objetivo rescatar la labor que ha realizado don Víctor Hugo en su vida como historiador y servir de fuente de información secundaria para futuras investigaciones.

La metodología del trabajo consistió en la revisión de distintos escritos elaborados por él, así como otros documentos que hablan sobre su obra. A cada documento se le hizo una descripción bibliográfica, se le adjuntó una lista de descriptores 
y se indicó la ubicación según las diferentes bibliotecas y centros de documentación visitados, siendo éstos: de la Universidad de Costa Rica, el Sistema de Bibliotecas, Documentación e Información (SIBDI); la Biblioteca Eugenio Fonseca Tortós; el centro de documentación del Centro de Investigaciones Históricas de América Central; el centro de documentación del Instituto de Investigaciones Sociales. De la Universidad Nacional de Costa Rica, la Biblioteca Joaquín García Monge; el centro de información documental y el fondo bibliográfico de historia de la Facultad de Ciencias Sociales. También se visitó la Biblioteca Nacional Miguel Obregón Lizano y la biblioteca de la Universidad Estatal a Distancia. Sin embargo, es importante mencionar que por responder esta investigación a los requisitos de un curso no fue posible cubrir todas las bibliotecas donde hay información escrita por el investigador objeto de nuestro estudio. Consideramos que para una futura investigación se recomienda contemplar las siguientes bibliotecas: la del Archivo Nacional, la de la Asamblea Legislativa, la del Museo Nacional, la de la Facultad Latinoamericana de Ciencias Sociales (FLACSO), entre otras; esto cobra importancia al considerar que don Víctor es una persona con muchos años por delante y que todavía tiene mucho por dar como científico e investigador social que es.

Además, se recuperó información a través de Internet, mediante buscadores, bases de datos y metabuscadores y del archivo en línea del periódico La Nación y del catálogo en línea de la Biblioteca del Congreso de los Estados Unidos.

La estructura de la biobibliografía es la siguiente: primero se presentan las instrucciones para el usuario, que consisten en la descripción detallada de la estructura de los diferentes tipos de registros que encontrará a través de este documento. Se sugiere al lector leer estas instrucciones antes de llevar a cabo cualquier búsqueda en este trabajo, pues esto, facilitará su primer acercamiento con la misma. Se incluye la lista de abreviaturas correspondientes a cada una de las unidades de información que se citan en la bibliografía.

La biografía del doctor Acuña Ortega se elaboró a partir de información suministrada por don Víctor, mediante una entrevista semiestructurada y un currículum facilitado por él a las autoras; también se contó con la valiosa colaboración del Doctor en Historia, señor David Díaz, quien no sólo es colega del biografiado, sino también su ex-alumno, suministrando información complementaria a la anterior y recopilada mediante una entrevista.

Por último se encuentra la bibliografía que incluye las obras de don Víctor Hugo Acuña Ortega, ordenada según el tipo de documento: libros, publicaciones periódicas, reuniones congresos o seminarios y, por último, material con formato no convencional; es decir, aquellos cuya forma no corresponde a ninguna de las categorías anteriores, por ejemplo, casetes, discos compactos y artículos bajados de internet. Muchos de los registros incluyen como anexo la fotografía o fotocopia de la portada o parte del documento a que se hace referencia. 


\section{INSTRUCCIONES PARA EL USUARIO}

Para aprovechar mejor este instrumento, es recomendable tener en cuenta los siguientes aspectos:

Este documento cuenta de dos partes:

1. La Biografía de don Víctor Hugo Acuña Ortega, que resume algunos acontecimientos en la vida de este autor, relacionados con su labor académica y de investigación.

2. La Bibliografía de don Víctor Hugo Acuña; que contempla una serie de registros destinados a identificar cada una de las obras de las que ha sido autor, editor o que tratan sobre él, desde la década de los años 70 al 2008.

Dentro de la Bibliografía, los registros se dividen a lo largo de la obra en cuatro categorías:

1. Monografías (libros, tesis, documentos inéditos, compilaciones);

2. Publicaciones periódicas (artículos de revista y artículos de periódicos);

3. Reuniones, congresos, conferencias y seminarios;

4. Documentos no convencionales (grabaciones sonoras) y

5. Documentos recuperados en Internet.

Dentro de dichas categorías los registros podrán encontrarse ordenados cronológica y alfabéticamente, de acuerdo al apellido del o los autores y título de la obra correspondiente. Sin embargo, se aconseja al usuario que busca un registro en específico, hacer uso de los diferentes Índices, que acompañan a esta obra y de los que posteriormente se tratará su forma de uso.

Los registros de la bibliografía incluyen los siguientes datos:

1. Número secuencial: número de orden al cual refieren los Índices de esta obra.

2. Notas: aclaraciones acerca del documento descrito. Este aspecto no se consigna en todos los registros.

3. Descriptores: términos que resumen el contenido de la obra descrita.

4. Ubicación: siglas de la unidad de información y signatura asignada a ese documento en ese lugar. 


\section{LISTA DE ABREVIATURAS}

\begin{tabular}{|c|c|}
\hline SIGLA & UNIDAD DE INFORMACIÓN \\
\hline BDDJN & Banco de Datos de Doctrina Jurídica Nacional \\
\hline BCMA & Biblioteca Carlos Monge Alfaro, Universidad de Costa Rica \\
\hline BEFT & Biblioteca Eugenio Fonseca Tortós, Universidad de Costa Rica \\
\hline BJGM & Biblioteca Joaquín García Monge, Universidad Nacional de Costa Rica \\
\hline BLDT & Biblioteca Luis Demetrio Tinoco, Universidad de Costa Rica \\
\hline $\mathrm{BN}$ & Biblioteca Nacional \\
\hline CIDCACS & $\begin{array}{l}\text { Centro de Investigación y Documentación Centroamericana en Ciencias } \\
\text { Sociales, Universidad de Costa Rica }\end{array}$ \\
\hline CIDCSO & $\begin{array}{l}\text { Centro de Información Documental de la Facultad de Ciencias Sociales, } \\
\text { Universidad Nacional de Costa Rica }\end{array}$ \\
\hline CIHAC & $\begin{array}{l}\text { Centro de Documentación del Centro de Investigaciones Históricas de } \\
\text { América Central, Universidad de Costa Rica }\end{array}$ \\
\hline CIHAC-CM & $\begin{array}{l}\text { Centro de Documentación del Centro de Investigaciones Históricas de } \\
\text { América Central - Colección Meléndez, Universidad de Costa Rica }\end{array}$ \\
\hline FBEH & Fondo Bibliográfico de Historia, Universidad Nacional de Costa Rica \\
\hline $\mathrm{LC}$ & Biblioteca del Congreso de los Estados Unidos de América \\
\hline MHJS & Museo Histórico Juan Santamaría \\
\hline
\end{tabular}




\begin{tabular}{|l|l|}
\hline UCR & Universidad de Costa Rica \\
\hline UNA & Universidad Nacional de Costa Rica \\
\hline UNED & Universidad Estatal a Distancia \\
\hline
\end{tabular}

\section{BIOGRAFÍA}

Don Víctor Hugo Acuña Ortega, nace en San José, un 6 de junio de 1949, hijo de Carmen Ortega Canales y Víctor Manuel Acuña García.

\section{ESTUDIOS Y TÍTULOS ACADÉMICOS}

Realizó sus estudios de educación secundaria en el Liceo de San José, en esta etapa de su vida le interesó el área de las letras, principalmente la literatura.

En 1967 entró a la Universidad de Costa Rica, donde ingresó a la carrera de Economía la cual no lo llegó a cautivar, trasladándose a estudiar Historia; encontrando en ella su verdadera vocación. Durante este período él, además de llevar los cursos de su carrera, cursó materias de otras áreas que le permitieron tener un mayor bagaje cultural, entre ellas filosofía y sociología; el ambiente de esta última lo enriquece más en lo social y en lo político.

Por otro lado, conoció diferentes personajes que lograron influenciarlo; cuando cursó Humanidades, conjunto de materias que se deben llevar al ingresar a la Universidad de Costa Rica, su profesora Rose Mary Karpinsky le despertó la inquietud por la historia. Otras personalidades que dejan huella en don Víctor Hugo son: su amigo y compañero Gastón Fournier Facio, Paulino González Villalobos, Jorge Enrique Guier, Francisco Amighetti y Hilda Chen Apuy.

Durante estos primeros años de universidad, llega a sus manos el libro La historia y las ciencias sociales de Fernand Braudel, destacado historiador de origen francés; quien le cambia la manera de ver la historia.

Además de lo mencionado anteriormente, cuando llega a Costa Rica el historiador brasileño Ciro Cardoso, ejerce influencia sobre don Víctor. Demuestra que es posible desarrollar otro tipo de historia, diferente a la memorística que imperaba en ese momento, y que todavía sigue dominando las aulas de la secundaria. Don 
Ciro dirige su tesis de licenciatura y lo estimula y apoya para optar para una beca e ir a estudiar a París.

A finales de 1974 viajó a París, donde estudió en la École de Hautes Études en Sciences Sociales; ahí permanece hasta mediados de 1978, año en que obtiene su título de Doctor en Historia. Durante este periodo, se desplaza entre Francia y España; en este último país trabajó en el Archivo de Indias en Sevilla para realizar su investigación, donde localiza el Informe del Consulado de Guatemala sobre las causas que tienen obstruido el comercio y los medios de renovarlas, 1978; documento transcrito por sus asistentes y publicado en el Anuario de Estudios Centroamericanos (vol. 9, p. 123-141, 1983).

A su regreso a Costa Rica, se incorpora como profesor asociado en la Escuela de Historia de la UCR.

\section{EJERCICIO PROFESIONAL}

A lo largo de su vida se ha desempeñado en diversas actividades y ha asumido diversos cargos, dentro y fuera de la Universidad de Costa Rica.

Entre los años 1978 al 2008 ha laborado como docente de la Escuela de Historia y del Posgrado Centroamericano en Historia, y luego se pensiona.

Además, ha sido profesor invitado en prestigiosas universidades del extranjero en diferentes momentos; ellas son: la Université François Rabelais de Tours, la Universidad de Burdeos III y la Universidad de Toulouse Le Mirail en Francia; la Universidad Nacional de Colombia, en su Sede en Medellín; la Escuela Nacional de Antropología e Historia en México; en el Posgrado de Ciencias Sociales de FLACSO en Guatemala; en el Instituto Universitario Ortega y Gasset, Madrid, España; en el Instituto Latinoamericano de la Universidad Libre de Berlín en Alemania; y además ha sido profesor visitante en el Saint Antony's College de la Universidad de Oxford, Inglaterra.

Sus actividades académicas no se limitan al cargo de profesor; dentro de la UCR, asumió también diferentes cargos administrativos: Subdirector del Centro de Investigaciones Históricas (1983-1985), al mismo tiempo que como Director del Posgrado Centroamericano en Historia. Director del Centro de Investigaciones Históricas desde 1985 a 1989. Entre 1994 y 1998 Director del Centro de Investigaciones Históricas de América Central.

Del 2001 al 2004 tiene bajo su responsabilidad el Programa Estado, Nación e Identidades en América Central del Centro de Investigaciones Históricas de América Central. En el 2004 coordina el Convenio de Cooperación entre el Instituto de Altos Estudios de América Latina de París (IHEAL) y la Universidad de Costa Rica. 
Y del 2004 al 2007 se desempeñó como Coordinador del Programa Historia y Estudios de Costa Rica y Nicaragua, Sede Regional de Guanacaste-Centro de Investigaciones Históricas de América Central, Liberia, Guanacaste.

Por otro lado, ha asumido funciones en diversas instituciones ajenas a la Universidad de Costa Rica, entre ellas fundaciones, revistas y editoriales. A manera de ejemplo:

- Miembro del jurado de las siguientes actividades:

- $\quad$ En 1986 de los Premios Nacionales.

- Premios Áncora, Costa Rica en los años 2001 y 2002.

- $\quad$ XI Muestra de Cine y Video, Costa Rica, en 2002.

- En los años 2004 y 2006, en Premios Nacionales en la Rama de Historia, Costa Rica.

- $\quad$ Cinergia, Costa Rica en 2007.

- Miembro de la junta directiva de las siguientes organizaciones y períodos:

- $\quad$ En 1984, Junta Directiva de la Editorial Costa Rica.

- 1995 hasta 1997 Presidente de la Asociación Pro Historia Centroamericana, Costa Rica.

- Del 2000 al 2007 en la Fundación Ars Teorética, Costa Rica.

- Además, de 1980 a 1988 participó en el Consejo Editorial de la Revista de Ciencias Sociales, Costa Rica

- De 1989-1990 Miembro de la Comisión Editorial de la Universidad de Costa Rica.

- $\quad$ En los períodos1985-1989, 1992-1997 y 2001-2006, miembro del Consejo Editorial de la Revista de Historia, Costa Rica.

- Ha realizado asesorías en instituciones, como la Coordinadora Educativa y Cultural Centroamericana (CECC), el Instituto Centroamericano de Administración Pública (ICAP), la Facultad Latinoamericana de Ciencias Sociales (FLACSO), el Museo Nacional de Costa Rica y el Centro de Estudios para la Acción Social (CEPAS), del que también fue fundador.

- También ha participado en diversos eventos científicos nacionales e internacionales, como simposios, conferencias, congresos, cursos, seminarios y encuentros. La siguiente lista da cuenta de los eventos en que ha participado:

- 1982: Seminario "El desarrollo del capitalismo en América Central (1870-1970)", Facultad de Ciencias Políticas y Sociales de la U.N.A.M.: México.

- 1984: Simposio "Historia, problemas y perspectivas agrarias en Costa Rica", Universidad Nacional, Heredia, Costa Rica.

- 1985: Congreso Internacional de Americanistas, Simposio sobre movimientos sociales: Bogotá. 
- 1985: Quinto Congreso de Historia de Colombia: Armenia.

- 1986: Simposio Internacional "La Costa Rica cafetalera: economía, sociedad y estructuras de poder", Universidad Nacional, Heredia Costa Rica.

- 1987: Sexto Congreso Internacional de Historia Oral: Oxford, Inglaterra.

- 1987: Segundo Seminario sobre Historia del Sindicalismo en América Latina,

- Clacso-Instituto Nacional de Antropología e Historia de México: Tlaxcala.

- 1988: Primer encuentro de historiadores orales de América Latina y España, Instituto Mora, México.

- 1989: Seminario "La enseñanza de la historia en América Latina", Georg-Eckert Institut, Braunschweig, Alemania.

- 1991: Primer encuentro de historiadores de Honduras, Tegucigalpa, (Invitado especial).

- 1992: Primer Congreso Centroamericano de Historia, Universidad Autónoma de Honduras, Tegucigalpa, (Invitado especial).

- 1993: Seminario "Balance histórico del estado-nación en América Central", CEMCA-FLACSO, San Salvador.

- 1993: Tenth Annual Latin American Labor History Conference, Duke University, Carolina del Norte, Estados Unidos, (comentarista).

- 1994: Segundo Congreso Centroamericano de Historia, Universidad de San Carlos, Guatemala.

- 1995: Seminario "América Latina frente al siglo XXI", Universidad de Rosario, Rosario, Argentina.

- 1996: Tercer Congreso Centroamericano de Historia, Universidad de Costa Rica, San José.

- 1997: Curso internacional de verano: "Centroamérica: paz, transición y gobernabilidad", Universidad de Extremadura, Jarandilla de la Vera.

- 1998: Cuarto Congreso Centroamericano de Historia, Universidad Autónoma de Nicaragua, Managua (Invitado especial).

- 2000: Quinto Congreso Centroamericano de Historia, Universidad de El Salvador, San Salvador.

- 2001: Seminario "Agendas Intelectuales en América Latina", IDESFundación Rockefeller, Buenos Aires.

- 2002: XIII Congreso de AHILA, Ponta Delgada, Sao Miguel, Islas Azores, Portugal.

- 2003: Primer Encuentro de Historia de El Salvador, San Salvador.

- 2003: Simposio "Historia, política, memoria", Universidad de Costa Rica, San José. 
- 2006: Conference on Latin American History, Reunión Anual de la American Historical Association, Philadelphia, Estados Unidos.

- 2006: Simposio Fútbol y Cultura: Alemania-Costa Rica, Universidad de Potsdam, Alemania.

- 2007: Simposio Internacional "Filibusterismo y Destino Manifiesto en las Américas", Liberia, Guanacaste.

- 2007: Primer Congreso sobre Cultura de Guanacaste, Santa Cruz, Guanacaste.

- En el año 2008 se acoge a su pensión.

\section{LABOR INVESTIGATIVA}

Muy importante es su amplia producción literaria, ha abordado diferentes áreas de la historia económica y social de Costa Rica y Centro América, reflejo de su pensamiento, con temas como: concepciones de la comunidad política durante la Independencia; diferentes versiones sobre la guerra contra los filibusteros; análisis de historiografía; el café; e incluso, análisis y comentarios a textos de otros autores, etc.

Según Acuña Ortega (2008, comentario personal) y Díaz Arias (2008, comentario personal), la producción investigativa de este científico social se puede analizar en cuatro etapas.

La primera etapa comprende la historia colonial y la historia económica del siglo XVIII. Este periodo lo vive entre sus estudios de licenciatura y doctorado.

La segunda, corresponde a su regreso de Europa, cuando se vincula con distintos intelectuales de diferentes disciplinas y establecen el Centro de Estudios para la Acción Social (CEPAS). Corre la década de los años 80, época muy convulsa en Centro América, por lo que don Víctor y su sensibilidad social se interna en la historia del siglo XIX y XX, principalmente este último y se inclina por una historia más social. Estudia y escribe sobre los trabajadores y grupos populares de Costa Rica y Centro América.

La tercera etapa, a mediados de la década de los 90, se interesa por la problemática de las identidades, incluso tiene varias ponencias sobre identidad nacional sin publicar.

Su cuarta y última etapa comprende desde hace unos cinco años, donde su interés ha estado marcado por el estudio de la memoria nacional. Busca la forma de hacer una historia política diferente.

Además, según Díaz Arias, ${ }^{4}$ don Víctor Hugo, durante su periodo como profesor, fue una fuente de ideas para sus alumnos; muchos de los temas de los trabajos finales de graduación del Posgrado en Historia, fueron sugeridos por este investigador. 
Su proyecto actual es poder dedicar tiempo al estudio del Estado no solo costarricense sino también del resto de los países centroamericanos. Su interés está centrado en el estudio de la historia cultural, la historia política y la historia social; es decir tratar de vincular la memoria, la identidad y el estado como un asunto de investigación.

Don Víctor no encuentra sentido en estudiar hoy día la historia patria, él habla más bien de una historia global, interrelacionada. Menciona que el desarrollar una historia nacional puede llegar a desencadenar mayor xenofobia en lugar de una mayor tolerancia e integración de las distintas culturas que hoy habitan Costa Rica.

\title{
RECONOCIMIENTOS
}

Su trayectoria le ha hecho merecedor también de múltiples reconocimientos, algunos de los más relevantes son: en 1986 es designado Catedrático en la UCR; gana una Beca de Investigación en 1989, para realizar una pasantía en el IberoAmerikanisches Institut de Berlín; la Beca Fulbright de Investigación en New Orleáns 1993; en ese mismo año recibe el Premio Nacional de Historia (Costa Rica). En 1998 recibe en España la Beca Intercampus del Instituto de Cooperación Iberoamericana. Grado de "Officier" a través de Palmas Académicas de la República Francesa en el 2004. Premio Aportes a la Creatividad de la Florida Ice and Farm, Costa Rica, en el 2005. Y la Beca de Investigación CONARE-DAAD, en Alemania, 2006

\section{BIBLIOGRAFÍA}

\author{
MONOGRAFÍAS
}

001

Acuña-Ortega, V.H. (1974). Historia económica del tabaco. Época colonial. (Tesis de Licenciatura en Historia). Universidad de Costa Rica. Facultad de Ciencias Sociales. Escuela de Historia y Geografía.

PRODUCCION; COMERCIO; MONOPOLIOS; COSECHAS; VENTAS; TABACO; HISTORIA; PERIODO COLONIAL; ECONOMIA; ASPECTOS SOCIALES

BEFT

BLDT

TFG-T 245

$\mathrm{BN}$

TFG 3137

C.R. 633.71 A189h 
002

Acuña-Ortega, V.H.; Guzmán-Stein, L.; Gutiérrez-Gurdián, H. (1976). Cómo preparar trabajos escritos. San José, C.R.: UCR, Facultad de Ciencias Sociales, Escuela de Trabajo Social.

NOTA: Existe reimpresión del año 1978

INVESTIGACION; METODOLOGÍA

BCMA

BEFT

Biblioteca Sede de Guanacaste

BJGM

$\mathrm{BN}$
029.6 G993c

$001.430 .18 \mathrm{G} 993 \mathrm{c}$

$029.6 \mathrm{G} 993 \mathrm{c}$

808.02 G993c

C.R. 378.17 G993c

003

Acuña-Ortega, V.H. (1978). Le commerce extérieur du Royaume de Guatemala au XVIII siècle 1700-1821: une étude structurelle. (Tesis de Doctorado). Université de Paris-Sorbonne (Paris IV). École des Hautes Études en Sciences Sociales.

HISTORIA ECONÓMICA; GUATEMALA; AMERICA CENTRAL; SIGLO XVIII; COMERCIO EXTERIOR

BLDT

$382 \mathrm{~A} 184 \mathrm{c}$

004

Acuña-Ortega, V.H. (1984). La huelga bananera de 1934. San José, C.R.: CENAP-CEPAS.

HUELGAS; HISTORIA

BEFT

BLDT

Biblioteca Sede de Guanacaste

Biblioteca Sede de Occidente

Biblioteca Sede de Limón

CIHAC.CM
$331.892 \mathrm{~A} 184 \mathrm{~h}$

$331.892 \mathrm{~A} 184 \mathrm{~h}$

$331.892 \mathrm{~A} 184 \mathrm{~h}$

$331.892 \mathrm{~A} 184 \mathrm{~h}$

$331.892 \mathrm{~A} 184 \mathrm{~h}$

E.177-62

005

Acuña-Ortega, V.H.(1984).Clases sociales y conflicto social en la economía cafetalera costarricense: productores contra beneficiadores (1932-1936). (Serie Avances de Investigación del Centro de Investigaciones Históricas. No. 10). San José, C.R.: UCR, CIHAC.

CLASES SOCIALES; CONFLICTOS SOCIALES; FORMACION DE CAPITAL; CAFE; 
PRODUCTORES DE CAFÉ

BCMA

BEFT

Biblioteca Sede de Occidente

CIDCSO

CIHAC
301.44 A184c

$323.3 \mathrm{~A} 184 \mathrm{c}$

$301.44 \mathrm{~A} 184 \mathrm{c}$

05.03.05 1066

006

Acuña-Ortega, V.H. (1985). Patrones de conflicto social en la economía costarricense: 1900-1948. [San José, C.R.]: [s. n.].

CAFE; PRODUCCION; ECONOMIA

BEFT

301 C7492pa

007

Acuña-Ortega, V.H. (1986). Cuestiones de memoria popular e historia social. En: Memoria y cultura popular costarricense. San José, C.R.: CENAP, URUK Editores,. (45-52).

CULTURA POPULAR - COSTA RICA; HISTORIA SOCIAL - COSTA RICA

BEFT

$306 \mathrm{C} 397 \mathrm{~m}$

008

Acuña-Ortega, V.H. (1986). Los orígenes de la clase obrera en Costa Rica: las huelgas de 1920 por jornada de ocho horas. San José, C.R.: CENAPCEPAS.

HUELGAS; CRISIS ECONOMICA; MOVIMIENTOS OBREROS; TRABAJADORES; CLASES SOCIALES; JORNADA DE TRABAJO

BEFT

CIHAC.CM
$331.892 \mathrm{~A} 184 \mathrm{o}$

E.175.48

009

Acuña-Ortega, V.H.; Molina-Jiménez, I. (1986). Desarrollo económico y social de Costa Rica: de la colonia a la crisis de 1930. San José, C.R.: Editorial Alma Máter.

HISTORIA ECONOMICA; DESARROLLO ECONOMICO Y SOCIAL; CRISIS ECONOMICA; HISTORIA

BEFT

BLDT

BN
$330.972 .86 \mathrm{~A} 184 \mathrm{~d}$

338.9 A $184 \mathrm{~d}$

C.R. 330.972.86 A184d 
Biblioteca Sede del Atlántico

Biblioteca Sede de Limón

Biblioteca Sede de Occidente

CIHAC.CM

UNED
338.9 A $184 d$

338.9 A $184 d$

338.9 A $184 d$

E. 175

$330.097 .286 \mathrm{~A} 189 \mathrm{~d}$

010

Acuña-Ortega, V.H. (1987). Una alternativa democrática para Costa Rica. Por E. Torres-Rivas. Costa Rica: crisis y desafíos. San José, C.R.: DEI. (181-190).

DEMOCRACIA; SISTEMAS DE GOBIERNO

CIDCACS

01869.01-16

011

Acuña-Ortega, V.H. (1987). La ideología de los pequeños y medianos productores cafetaleros costarricenses (1900-1961). (Serie Avances de Investigación del Centro de Investigaciones Históricas. No. 23). San José, C.R.: UCR, CIHAC.

Nota: la BN lo archiva dentro de la colección de revistas.

PRODUCTORES; CAFE; IDEOLOGIAS; HISTORIAECONOMICA; SOCIEDAD,CONCIENCIA DE CLASE, LUCHA DE CLASES

BEFT

BLDT

$\mathrm{BN}$

CIDCACS

CIHAC
$305.56 \mathrm{~A} 184 \mathrm{i}$

$338.1 \mathrm{~A} 184 \mathrm{i}$

H 972.8605 A946a C.R.

05.03 .021067

012

Acuña-Ortega, V.H. [1989]. La historia oral, las historias de vida y las ciencias sociales. En: [Historia: teoría y métodos]. [San José, C.R.: EDUCA]. (225-261).

Nota: fotocopia

CIENCIAS SOCIALES; HISTORIA; METODOLOGIA HISTORICA; METODO HISTORICO; HISTORIA DE VIDA; FUENTES ORALES; HISTORIA ORAL

CIDCSO

014224

013 
Acuña-Ortega, V.H. (1990). Democracia y cultura política en Costa Ric. En: Democracia y cultura política en Costa Rica. San José, C.R.: Ministerio de Cultura Juventud y Deportes, 1990. (155-162).

COSTA RICA - POLITICA; DEMOCRACIA; CULTURA POLÍTICA

CIDCACS

04.02.02 D383d

014

Acuña-Ortega, V.H.; Molina-Jiménez, I. (1991). Historia económica y social de Costa Rica (17501950). (Serie Colección Debate ). San José, C.R.: Editorial Porvenir.

ECONOMIA; HISTORIA ECONOMICA; HISTORIA SOCIAL; CAPITALISMO; CLASES SOCIALES; LUCHA DE CLASES; CONFLICTOS SOCIALES; SINDICATOS; DESARROLLO AGROPECUARIO; PERIODO COLONIAL; DEMOCRACIA; OBREROS INDUSTRIALES; SINDICATOS; HISTORIA CULTURAL

\section{BEFT}

BLDT

Biblioteca de Derecho

Biblioteca Sede del Atlántico

Biblioteca Sede de Guanacaste

Biblioteca Sede de Occidente

BDDJN

BJGM

$\mathrm{BN}$

CIHAC

CIHAC.CM
330.972.86 A184h

$338.9 \mathrm{~A} 184 \mathrm{~h}$

$338.9 \mathrm{~A} 184 \mathrm{~h}$

338.9 A $184 \mathrm{~h}$

$338.9 \mathrm{~A} 184 \mathrm{~h}$

338.9 A $184 \mathrm{~h}$

$338.9 / \mathrm{A} 184 \mathrm{~h}$

330.972.86 A184h

C.R. 330.972.86 A184h

05.01.01 2665 Hall

E.175

015

Acuña-Ortega, V.H. (1991). Conflicto y reforma en Costa Rica: 1940-1949. (Serie Nuestra Historia, No. 17). San José, C.R.: EUNED.

NOTA: Existe reimpresión del año 1992 y de 1993

HISTORIA; ESTADO; CRISIS POLITICA; LIBERALISMO; ADMINISTRACION CALDERON GUARDIA; ELECCIONES; REFORMA

$\begin{array}{ll}\text { BCMA } & 972.860 .534 \text { A184c } \\ \text { BEFT } & 01.04 .037 \text { FAS 17 } \\ \text { BJGM } & 972.86 \text { A184c } \\ \text { BN } & \text { C.R. } 972.86 \text { A1846c } \\ \text { Biblioteca Sede de Occidente } & 972.860 .534 \text { A184c } \\ \text { Biblioteca Sede de Guanacaste } & 972.860 .534 \text { A184c } \\ \text { CIHAC } & \text { E246-17 } \\ \text { CIHAC } & \text { E246 }\end{array}$


$\mathrm{LC}$

UNED
F1547.5 .A38 1991

972.86 A189c-1992

016

Acuña-Ortega, V.H. (1992). Artesanos, obreros urbanos y proletarios de enclaves en Centroamérica en el período liberal: una minoría activa. (Serie Avances de Investigación del Centro de Investigaciones Históricas. No. 59). San José, C.R.: UCR, CIHAC.

Nota: la BN y la BJGM lo archiva dentro de la colección de revistas.

HISTORIA; TRABAJADORES; ZONAS URBANAS; ARTESANOS; OBREROS INDUSTRIALES; ASPECTOS SOCIALES; ASPECTOS POLITICOS; SINDICATOS

BEFT
BLDT
BJGM
BN
Biblioteca Recinto de Tacares
Biblioteca Sede del Atlántico
Biblioteca Sede de Guanacaste
Biblioteca Sede de Occidente
Biblioteca Sede del Pacífico
CIHAC

BEFT

BJGM

BN

Biblioteca Recinto de Tacares

Biblioteca Sede del Atlántico

Biblioteca Sede de Guanacaste

Biblioteca Sede del Pacífico

CIHAC

972.8 A184a
331.790.9 A-184
H 900.72 A946a
H 972.8605 A946a C.R.
331.790.9 A-184a
331.790.9 A-184a
331.790.9 A-184a
331.790.9 A-184a
331.790.9 A-184a
05.03.05 1056 c3

017

Acuña-Ortega, V.H. (1993). Comentario [sobre Costa Rica: virtudes y vicios de una democracia 'perfecta' de Daniel Camacho M.]. Por R. Steichen-Jung y V.H. Acuña-Ortega. Democracia y democratización en Centroamérica. San José, C.R.: Editorial de la UCR, 1993. (237240).

DEMOCRACIA;CRITICADECONTENIDO;CAMACHOMONGE,DANIEL;METODOLOGIA; HISTORIA POLITICA; COSTA RICA

BEFT
BJGM
BN
CIHAC.CM-E
CIDCSO
LC

018

Acuña-Ortega, V.H. (1993). Nación y clase obrera en Centroamérica en la época liberal: 18701930. (Serie Avances de Investigación del Centro de Investigaciones Históricas. No. 66). San José, C.R.: UCR, CIHAC.

Nota: la BN lo archiva dentro de la colección de revistas. 
PROBLEMAS SOCIALES; POBREZA; CONDICIONES DE VIDA; RECESION ECONOMICA; ESTRATEGIA DEL DESARROLLO; TRABAJADORES; ANALISIS HISTORICO; CLASES SOCIALES

$\begin{array}{ll}\text { BEFT0 } & 4.03 .01506 \\ \text { CIDCACS } & 11469.00 \\ \text { CIHAC } & 04.03 .01506 \mathrm{c} 2 \\ \text { BN } & \end{array}$

019

Acuña-Ortega, V.H. (1993). Nación y clase obrera en Centroamérica en la época liberal, 18701930. San José, C.R.: UCR, CESPO.

PROBLEMAS SOCIALES; POBREZA; CONDICIONES DE VIDA; RECESION ECONOMICA; ESTRATEGIA DEL DESARROLLO; TRABAJADORES; ANALISIS HISTORICO; CLASES SOCIALES

\section{BLDT}

Biblioteca Recinto de Tacares

Biblioteca Sede del Atlántico

Biblioteca Sede de Guanacaste

Biblioteca Sede del Pacífico

$$
\begin{aligned}
& 331.09 \text { A184n } \\
& 331.09 \text { A184n } \\
& 331.09 \text { A184n } \\
& 331.09 \text { A184n } \\
& 331.09 \text { A184n }
\end{aligned}
$$

020

Acuña-Ortega, V.H., ed. (1993). Las repúblicas agroexportadoras: 1870-1945. Por V.H. AcuñaOrtega, (Ed.). Historia General de Centro América (Vol. IV). Madrid: FLACSO; Comunidad Económica Europea.

HISTORIA; CAFE; SOCIEDAD; TRABAJO; TENENCIA DE LA TIERRA; CRECIMIENTO ECONOMICO; COMERCIO EXTERIOR; CULTIVOS; BANANOS; COMERCIALIZACION; LIBERALISMO; PODER POLITICO; SOBERANIA; DEMOCRACIA; MOVIMIENTOS SOCIALES; CRISIS; ECONOMIA; MINORIAS ETNICAS; DICTADURA; GUERRA MUNDIAL

$\begin{array}{ll}\text { BEFT } & 01.04 .03610 \mathrm{v} .4 \\ \text { CIHAC } & 05.01 .01613 \\ \text { CIHAC.CM } & \text { E.134 } \\ \text { LC } & \text { F1436.H57 } 1993\end{array}$

021

Acuña-Ortega, V.H. (1993). Las clases subalternas y movimientos sociales en Centroamérica (18701930). Por V.H. Acuña-Ortega, (Ed.). Historia General de Centro América (Vol. IV). Madrid: FLACSO; Comunidad Económica Europea. (255-323).

HISTORIA; CAFE; SOCIEDAD; TRABAJO; TENENCIA DE LA TIERRA; CRECIMIENTO ECONOMICO; COMERCIO EXTERIOR; CULTIVOS; BANANOS; COMERCIALIZACION; 
LIBERALISMO; PODER POLITICO; SOBERANIA; DEMOCRACIA; MOVIMIENTOS SOCIALES; CRISIS; ECONOMIA; MINORIAS ETNICAS; DICTADURA; GUERRA MUNDIAL

$\begin{array}{ll}\text { BEFT } & 01.04 .03610 \mathrm{v} .4 \\ \text { CIHAC } & 05.01 .01613 \\ \text { CIHAC.CM } & \text { E.134 } \\ \text { LC } & \text { F1436.H57 } 1993\end{array}$

022

Acuña-Ortega, V.H. (1994). Historia del vocabulario político en Costa Rica: Estado, República, Nación y Democracia (1821-1949). (Serie Avances de Investigación. No. 75). San José, C.R.: UCR, CIHAC.

HISTORIA; POLITICA; VOCABULARIOS; LENGUAJE

BCMA

$320.03 \mathrm{~A} 184 \mathrm{H}$

BEFT

$05.01 .01 \mathrm{HI}-1568$

CIHAC

$04.04 .021180 \mathrm{c} 2$

023

Goldenberg, O.; Acuña-Ortega, V.H. (1994). Género en la informalidad: historias laborales centroamericanas. San José, C.R.: FLACSO.

GÉNERO; HISTORIAS DE VIDA; MUJERES, HOMBRES; DESARROLLO SOCIAL; PARTICIPACION DE LA MUJER; ROL DE LA MUJER; TRABAJO; SECTOR INFORMAL

BEFT
BLDT
BJGM
BN
CIDCACS
LC

024

Acuña-Ortega, V.H. (1995). Autoritarismo y democracia en Centroamérica: la larga duración, Siglos XIX y XX. Por K.D. Tangermann. Ilusiones y dilemas: la democracia en Centroamérica. San José, C.R.: FLACSO, 1995. (63-97).

DEMOCRACIA - AMERICA CENTRAL; AUTORITARISMO; HISTORIA; HISTORIA POLITICA; SIGLO XIX; SIGLO XX; ENSAYO; CLASES DOMINANTES; INSTITUCIONES POLITICAS; SISTEMA POLITICO

CIDCACS
14.02 .03822

331.216 G618g

$331.216 \mathrm{G} 618 \mathrm{~g}$

C.R. $331 \mathrm{G} 618 \mathrm{~g}$

16204.00 FLACSO

HD6060.65.C35 G65 1994 
025

Acuña-Ortega, V.H. (1995). Historia del vocabulario político costarricense: Estado, República, Nación y Democracia (1821-1949). Por A. Taracena-Arriola y J. Piel. Identidades nacionales y estado moderno en Centroamérica. San José, C.R.: Editorial Universidad de Costa Rica, 1995.

CIENCIAS POLITICAS; GLOSARIOS, VOCABULARIOS, ETC.; COSTA RICA; POLITICA Y GOBIERNO

BEFT

CIHAC

CIHAC-CM
$05.01 .01 \mathrm{HI}-1371$

05.01.01 956 TARA

E-173

026

Vannini, M. (Ed.). (1995). Encuentros con la historia. Managua, Nicaragua: Instituto de Historia de Nicaragua, Universidad Centroamericana: Centro Francés de Estudios Mexicanos y Centroamericanos.

Nota: la descripción del libro indica que incluye documentos sobre historia y/o sobre el método histórico elaborado por Víctor Hugo Acuña Ortega, entre otros autores o colaboradores.

AMERICA CENTRAL; NICARAGUA; HISTORIA; CONGRESOS; ACUÑA ORTEGA, VICTOR HUGO

LC

F1422

027

Acuña-Ortega, V.H.; Sibaja-Chacón, L.F. (1996). Estudios Sociales 5: texto. (Serie Hacia el siglo XXI). San José, C.R.: Editorial Universidad de Costa Rica : PROMECE, Ministerio de Educación Pública, 1996.

ESTUDIOS SOCIALES; HISTORIA; ENSEÑANZA

BCMA

Biblioteca Sede de Occidente

CIHAC.CM
372.83 A184E 1996

$372.83 \mathrm{~A} 184 \mathrm{e}$

E.246

028

Acuña-Ortega, V.H.; Sibaja-Chacón, L.F. (1996). Estudios Sociales 5: guía didáctica. (Serie Hacia el siglo XXI.). San José, C.R.: Editorial Universidad de Costa Rica : PROMECE, Ministerio de Educación Pública.

Nota: existe reimpresión del año 1997.

ESTUDIOS SOCIALES; ENSEÑANZA 
BCMA

$\mathrm{BN}$

Biblioteca Sede de Occidente

UNED
372.83 A184es

C.R. 372.83 A184et V

372.83 A184es

$372.830 .44 \mathrm{~A} 184 \mathrm{e}$

029

Acuña-Ortega, V.H.;Sibaja-Chacón, L.F. (1996). Estudios Sociales 5: actividades. (Serie Hacia el siglo XXI). San José, C.R.: Editorial de la Universidad de Costa Rica : PROMECE, Ministerio de Educación Pública.

ESTUDIOS SOCIALES -ESTUDIO Y ENSEÑANZA; ESTUDIOS SOCIALES -PREGUNTAS Y RESPUESTAS; ESTUDIOS SOCIALES - ENSEÑANZA

BCMA

$\mathrm{BN}$

Biblioteca Sede de Occidente

UNED
372.83 A184et

C.R. 372.83 A184es V

372.83 A184et

372.830.44 A184e

030

Enríquez-Solano, F.; Acuña-Ortega, V.H.; Badilla-Gómez, P.; Fonseca-Corrales, E. (1996). Estudios Sociales 2: texto. (Serie hacia el siglo XXI). San José, C.R.: Editorial de la Universidad de Costa Rica : PROMECE, Ministerio de Educación Pública.

ESTUDIOS SOCIALES; ENSEÑANZA

$\mathrm{BN}$ C.R. 372.83 E821es II

031

Acuña-Ortega, V.H.;Sibaja-Chacón, L.F. (1997). Estudios Sociales 5: texto. (Serie Hacia el siglo XXI). San José, C.R.: Editorial Universidad de Costa Rica : PROMECE, Ministerio de Educación Pública.

ESTUDIOS SOCIALES; ENSEÑANZA

Biblioteca Sede de Occidente

$372.83 \mathrm{~A} 184 \mathrm{e}$

032

Acuña-Ortega, V.H.; Avendaño-Flores, I.; Botey-Sobrado, A.M.; Granados-Chaverri, C.; SolórzanoFonseca, J.C. (1997). Estudios Sociales 9: texto. (Serie Hacia el siglo XXI). San José, C.R.: Editorial de la Universidad de Costa Rica : PROMECE, Ministerio de Educación Pública.

ESTUDIOS SOCIALES; COSTA RICA - HISTORIA - ESTUDIO Y ENSEÑANZA

Biblioteca Sede de Occidente

300.7 E82et 
033

Acuña-Ortega, V.H.(1998). Memoria, olvido, impunidad y secularización política. Por J. Oyamburu; M.A. González-Muñiz; A. Jiménez. La percepción de lo político en Costa Rica. Heredia, C.R.: Editorial Fundación UNA. (211-220).

CRISIS POLITICA; MARCO INSTITUCIONAL; SOCIEDAD CIVIL; DEMOCRACIA; POLITICA

CIDCACS

$13753.01-22$

034

Coordinación Educativa y Cultural Centroamericana. Historia del istmo centroamericano: tomo I. [México]: Grupo Editorial Siquisirí, 2000.

Nota: Víctor Hugo Acuña Ortega es el coordinador académico por Costa Rica

AMERICA CENTRAL; HISTORIA; ESTUDIOS SOCIALES; ENSEÑANZA; ACUÑA ORTEGA, VICTOR HUGO

$\mathrm{BN}$

C.R. $972.8 \mathrm{H} 6732 \mathrm{~h}$

035

Acuña-Ortega, V.H. (2004). Capítulo 4: Historia republicana (1821-1930). Por Coordinación Educativa y Cultural Centroamericana. Guía temática y didáctica del libro de texto: historia del istmo centroamericano. México: Grupo Editorial Siquisirí. (211-236).

AMERICA CENTRAL; HISTORIA; ESTUDIOS SOCIALES; ENSEÑANZA

$\mathrm{BN}$

C.R. 972.8 C778g

036

Acuña-Ortega, V.H. (2004). Costa Rica en el siglo XX. Por Molina-Jiménes, I. y otros. Balances del siglo XX: historia, microbiología, medicina y física. (Serie Cuadernos de Historia de la cultura. No. 11). San José, C.R.: Editorial de la UCR. (11-19).

Nota: texto de una intervención en una tertulia de "El Farolito" (Centro Cultural Español), celebrada en octubre de 1999.

COSTA RICA; SIGLO XX; HISTORIA

BCMA

303.483 B171b 
037

Acuña-Ortega, V.H. (2004). The formation of the urban middle sectors in El Salvador, 19101944. Por A. Lauria-Santiago y L. Binford. Landscapes of struggle: politics, society, and community in El Salvador. Pittsburgh: University of Pittsburgh Press. (39-[49?]).

EL SALVADOR; HISTORIA; SIGLO XX; CONDICIONES SOCIALES; CLASES SOCIALES; LUCHA DE CLASES

LC

F1488 .L36 2004

038

Acuña-Ortega, V.H. (2005). Capítulo 19: Tiempo histórico y ciencia social en Centroamérica en la segunda mitad del siglo XX. Por Wallerstein, I. y otros. Espacio y tiempo en gestión $y$ análisis social. Conceptos emergentes y modelos de trabajo = Space and time in management and social análisis. Emerging concepts and working models. San José: UCR, CICAP. (12).

HISTORIA; CIENCIAS SOCIALES, CAMBIO SOCIAL; AMERICA CENTRAL; ANALISIS HISTORICO; ANALISIS DE CONTENIDO; TEORIA SOCIAL; ANTOLOGIA

$\begin{array}{ll}\text { CIDCACS } & 05.01 .01 \mathrm{E} 77 \mathrm{e} \\ \text { FBEH } & 038299\end{array}$

039

Acuña-Ortega, V.H. (2007). Historia e incertidumbre. (Serie Cuadernos de Historia de la Cultura, No. 22). San José, C.R.: Editorial Universidad de Costa Rica.

HISTORIA; SOCIOLOGIA; SIGLO XX; CIUDADANIA; ENSAYOS; SIGLO XXI; PODER

BCMA

BEFT

BN

Biblioteca Sede del Atlántico

Biblioteca Sede de Guanacaste

Biblioteca Sede de Limón

Biblioteca Sede de Occidente

Biblioteca Sede del Pacífico
$972.860 .52 \mathrm{~A} 184 \mathrm{~h}$

01.04 .0317386

C.R. 972.8605 A1846h

972.860.52 A184h

$972.860 .52 \mathrm{~A} 184 \mathrm{~h}$

972.860 .52 A184h

972.860.52 A184h

972.860.52 A184h

040

Acuña-Ortega, V.H. (2007). Estudiar historia. Por V.H. Acuña-Ortega. Historia e incertidumbre. (Serie Cuadernos de Historia de la Cultura. No. 22). San José, C.R.: Editorial Universidad de Costa Rica. (3-15).

HISTORIA; SOCIOLOGIA; SIGLO XX; CIUDADANIA; ENSAYOS; SIGLO XXI; PODER 
BCMA

BEFT

BN

Biblioteca Sede del Atlántico

Biblioteca Sede de Guanacaste

Biblioteca Sede de Limón

Biblioteca Sede de Occidente

Biblioteca Sede del Pacífico
972.860.52 A184h

01.04 .0317386

C.R. 972.8605 A1846h

$972.860 .52 \mathrm{~A} 184 \mathrm{~h}$

$972.860 .52 \mathrm{~A} 184 \mathrm{~h}$

972.860 .52 A184h

972.860.52 A184h

$972.860 .52 \mathrm{~A} 184 \mathrm{~h}$

041

Acuña-Ortega, V.H. (2007). Sudando con Clío. Por V.H. Acuña-Ortega. Historia e incertidumbre. (Serie Cuadernos de Historia de la Cultura. No. 22). San José, C.R.: Editorial Universidad de Costa Rica. (17-24).

HISTORIA; SOCIOLOGIA; SIGLO XX; CIUDADANIA; ENSAYOS; SIGLO XXI; PODER

BCMA

BEFT

BN

Biblioteca Sede del Atlántico

Biblioteca Sede de Guanacaste

Biblioteca Sede de Limón

Biblioteca Sede de Occidente

Biblioteca Sede del Pacífico
972.860.52 A184h

01.04 .0317386

C.R. 972.8605 A1846h

$972.860 .52 \mathrm{~A} 184 \mathrm{~h}$

$972.860 .52 \mathrm{~A} 184 \mathrm{~h}$

972.860 .52 A184h

$972.860 .52 \mathrm{~A} 184 \mathrm{~h}$

$972.860 .52 \mathrm{~A} 184 \mathrm{~h}$

042

Acuña-Ortega, V.H. (2007). Los desafíos de la historia en Centroamérica. Por V.H. Acuña-Ortega. Historia e incertidumbre. (Serie Cuadernos de Historia de la Cultura. No. 22). San José, C.R.: Editorial Universidad de Costa Rica. (25-37).

HISTORIA; SOCIOLOGIA; SIGLO XX; CIUDADANIA; ENSAYOS; SIGLO XXI; PODER

BCMA

BEFT

$\mathrm{BN}$

Biblioteca Sede del Atlántico

Biblioteca Sede de Guanacaste

Biblioteca Sede de Limón

Biblioteca Sede de Occidente

Biblioteca Sede del Pacífico
972.860.52 A $184 \mathrm{~h}$

01.04 .0317386

C.R. 972.8605 A1846h

972.860.52 A184h

972.860.52 A184h

$972.860 .52 \mathrm{~A} 184 \mathrm{~h}$

972.860.52 A184h

972.860.52 A184h

043

Acuña-Ortega, V.H.(2007).Historia y ciudadanía. Por V.H. Acuña-Ortega. Historia e incertidumbre. (Serie Cuadernos de Historia de la Cultura. No. 22). San José, C.R.: Editorial Universidad de Costa Rica. (39-54). 
HISTORIA; SOCIOLOGIA; SIGLO XX; CIUDADANIA; ENSAYOS; SIGLO XXI; PODER

BCMA

BEFT

BN

Biblioteca Sede del Atlántico

Biblioteca Sede de Guanacaste

Biblioteca Sede de Limón

Biblioteca Sede de Occidente

Biblioteca Sede del Pacífico
972.860.52 A184h

01.04 .0317386

C.R. 972.8605 A1846h

972.860.52 A184h

$972.860 .52 \mathrm{~A} 184 \mathrm{~h}$

972.860 .52 A184h

972.860.52 A184h

972.860.52 A184h

044

Acuña-Ortega, V.H. (2007). Historia e incertidumbre. Por V.H. Acuña-Ortega. Historia $e$ incertidumbre. (Serie Cuadernos de Historia de la Cultura. No. 22). San José, C.R.: Editorial Universidad de Costa Rica. (55-65).

HISTORIA; SOCIOLOGIA; SIGLO XX; CIUDADANIA; ENSAYOS; SIGLO XXI; PODER

BCMA

BEFT

BN

Biblioteca Sede del Atlántico

Biblioteca Sede de Guanacaste

Biblioteca Sede de Limón

Biblioteca Sede de Occidente

Biblioteca Sede del Pacífico
$972.860 .52 \mathrm{~A} 184 \mathrm{~h}$

01.04 .0317386

C.R. 972.8605 A1846h

972.860.52 A184h

$972.860 .52 \mathrm{~A} 184 \mathrm{~h}$

$972.860 .52 \mathrm{~A} 184 \mathrm{~h}$

$972.860 .52 \mathrm{~A} 184 \mathrm{~h}$

$972.860 .52 \mathrm{~A} 184 \mathrm{~h}$

045

Acuña-Ortega, V.H. (2007). Memoria, olvido, impunidad y secularización política. Por V.H. AcuñaOrtega. Historia e incertidumbre. (Serie Cuadernos de Historia de la Cultura. No. 22). San José, C.R.: Editorial Universidad de Costa Rica. ( 67-73).

HISTORIA; SOCIOLOGIA; SIGLO XX; CIUDADANIA; ENSAYOS; SIGLO XXI; PODER

BCMA

BEFT

BN

Biblioteca Sede del Atlántico

Biblioteca Sede de Guanacaste

Biblioteca Sede de Limón

Biblioteca Sede de Occidente

Biblioteca Sede del Pacífico
972.860.52 A184h

01.04 .0317386

C.R. 972.8605 A1846h

972.860.52 A184h

972.860.52 A184h

$972.860 .52 \mathrm{~A} 184 \mathrm{~h}$

$972.860 .52 \mathrm{~A} 184 \mathrm{~h}$

972.860.52 A184h

046

Acuña-Ortega, V.H. (2007). Retos y perspectivas del nuevo milenio. Por V.H. Acuña-Ortega. 
Historia e incertidumbre. (Serie Cuadernos de Historia de la Cultura. No. 22). San José, C.R.: Editorial Universidad de Costa Rica. 2007. (75-80).

HISTORIA; SOCIOLOGIA; SIGLO XX; CIUDADANIA; ENSAYOS; SIGLO XXI; PODER

BCMA

BEFT

BN

Biblioteca Sede del Atlántico

Biblioteca Sede de Guanacaste

Biblioteca Sede de Limón

Biblioteca Sede de Occidente

Biblioteca Sede del Pacífico
972.860.52 A184h

01.04 .0317386

C.R. 972.8605 A1846h

$972.860 .52 \mathrm{~A} 184 \mathrm{~h}$

$972.860 .52 \mathrm{~A} 184 \mathrm{~h}$

$972.860 .52 \mathrm{~A} 184 \mathrm{~h}$

972.860.52 A184h

$972.860 .52 \mathrm{~A} 184 \mathrm{~h}$

047

Acuña-Ortega, V.H. (2007). El lugar de la identidad y la identidad en su lugar. Por V.H. AcuñaOrtega. Historia e incertidumbre. (Serie Cuadernos de Historia de la Cultura. No. 22). San José, C.R.: Editorial Universidad de Costa Rica. 2007. (81-89).

HISTORIA; SOCIOLOGIA; SIGLO XX; CIUDADANIA; ENSAYOS; SIGLO XXI; PODER

BCMA

BEFT

BN

Biblioteca Sede del Atlántico

Biblioteca Sede de Guanacaste

Biblioteca Sede de Limón

Biblioteca Sede de Occidente

Biblioteca Sede del Pacífico
972.860.52 A184h

01.04 .0317386

C.R. 972.8605 A1846h

972.860.52 A184h

$972.860 .52 \mathrm{~A} 184 \mathrm{~h}$

$972.860 .52 \mathrm{~A} 184 \mathrm{~h}$

$972.860 .52 \mathrm{~A} 184 \mathrm{~h}$

$972.860 .52 \mathrm{~A} 184 \mathrm{~h}$

048

Acuña-Ortega, V.H.(2007). Elogio del inmigrante. Por V.H.Acuña-Ortega. Historia e incertidumbre . (Serie Cuadernos de Historia de la Cultura. No. 22). San José, C.R.: Editorial Universidad de Costa Rica. (91-100).

INMIGRANTES; CULTURA; HISTORIA; MIGRACION; CONFERENCIAS

BCMA

BEFT

$\mathrm{BN}$

Biblioteca Sede del Atlántico

Biblioteca Sede de Guanacaste

Biblioteca Sede de Limón

Biblioteca Sede de Occidente

Biblioteca Sede del Pacífico
972.860.52 A184h

01.04 .0317386

C.R. 972.8605 A1846h

972.860.52 A184h

$972.860 .52 \mathrm{~A} 184 \mathrm{~h}$

972.860 .52 A184h

972.860.52 A184h

972.860.52 A184h 
049

Acuña-Ortega, V.H. [s.f.]. Autoritarismo y democracia en Centroamérica: la larga duración (Siglos XIX y XX). San José: UCR, CIHAC.

PAISES EN DESARROLLO; PODER POLITICO; SISTEMAS POLITICOS; CONDICIONES DE VIDA; DICTADURA; DEMOCRACIA; AUTORITARISMO; DERECHOS HUMANOS; VIOLENCIA; GUERRA CIVIL; PARTICIPACION SOCIAL; ANALISIS HISTORICO; CORRUPCION; CLASES SOCIALES

CIDCACS

12499.00

050

Acuña-Ortega, V.H. [s.f.]. Clases sociales y conflicto social en la economía cafetalera costarricense, productores contra beneficiadores (1932-1936). Por C.L. Fallas-Monge; V.H. AcuñaOrtega, Movimientos sociales en Costa Rica. [s.1. : s.n.].

MOVIMIENTOS SOCIALES; MOVIMIENTOS OBREROS; CLASES SOCIALES; CONFLICTOS SOCIALES; HISTORIA; ECONOMIA AGRICOLA

BEFT

05.03 .073363

051

Acuña-Ortega, V.H. [s.f.]. La política en el trasiego de los restos y los restos de una política. [s.1. : s.n.].

HISTORIA; POLITICA

CIDCACS

02198.00

052 Acuña-Ortega, V.H. . [s.f.]. Patrones del conflicto social en el agro costarricense (siglos XVIIIXX). [San José, C.R: s.n.].

Nota: material mimeografiado

COSTA RICA; HISTORIA; AGRICOLA; AGRICULTURA; PROPIEDAD DE LA TIERRA

FBEH

HISTORIA 038103

053

Acuña-Ortega, V.H. [s.f.]. Vertientes del recuerdo. Historia y memoria de la guerra contra los filibusteros: Estados Unidos, Nicaragua y Costa Rica (siglos XIX-XXI). San José, C.R.: El autor.

Nota: documento inédito 
HISTORIA; CAMPAÑA NACIONAL; FILIBUSTEROS; ESTADOS UNIDOS; NICARAGUA; COSTA RICA

MHJS

\section{PUBLICACIONES PERÍODICAS}

ARTÍCULOS DE PERIÓDICO

054

Acuña-Ortega, V.H. (1970, 26 de octubre). ¿Qué es el Neorrealismo? Semanario Universidad, 4.

CINE; NEORREALISMO - ANTECEDENTES; NEORREALISMO - CARACTERÍSTICAS;

NEORREALISMO - REPRESENTANTES

BLDT

$378 \mathrm{U}$

055 Acuña-Ortega, V.H. (1970, 9 de noviembre). Chicago, Chicago. Semanario Universidad, 2.

CRITICA DE CINE

BLDT

$378 \mathrm{U}$

056

Acuña-Ortega, V.H. (1970, 23 de noviembre). David Copperfield. Semanario Universidad, 2.

CRITICA DE CINE; COPPERFIELD, DAVID

BLDT

$378 \mathrm{U}$

057

Acuña-Ortega, V.H. (1970, 7 de diciembre). IF. Semanario Universidad, 2.

CRITICA DE CINE; ANDERSON, LINDSAY; FREE CINEMA

BLDT

$378 \mathrm{U}$

058

Acuña-Ortega, V.H. (1971, 18 de enero). Oh! Qué bella guerra. Semanario Universidad, 2.

CRITICA DE CINE; CINE INGLÉS 
059

Acuña-Ortega, V.H. (1971, 15 de febrero). Z. Semanario Universidad, 2.

CRITICA DE CINE

BLDT

$378 \mathrm{U}$

060

Acuña-Ortega, V.H. (1971, 8 de marzo). Ingmar Bergman. Semanario Universidad, 2.

BERGMAN, INGMAR - OBRA; CINE SUECO; REVOLUCIÓN CINEMATOGRÁFICA

BLDT

$378 \mathrm{U}$

061

Acuña-Ortega, V.H. (1971, 15 de marzo). Ingmar Bergman II. Semanario Universidad, 2.

BERGMAN, INGMAR - OBRA; CINE SUECO

BLDT

$378 \mathrm{U}$

062

Acuña-Ortega, V.H. (1971, 22 marzo). Ingmar Bergman III. Semanario Universidad, 2.

BERGMAN, INGMAR - OBRA; CINE SUECO

BLDT

$378 \mathrm{U}$

063

Acuña-Ortega, V.H. (1971, 12 de abril). El montaje cinematográfico. Semanario Universidad, 2.

CINE; MONTAJE CINEMATOGRÁFICO; ELEMENTOS DEL FILM

BLDT

$378 \mathrm{U}$

064

Acuña-Ortega, V.H. (1971, 19 de abril). El montaje II. Semanario Universidad, 2.

CINE; MONTAJE CINEMATOGRÁFICO 
065

Acuña-Ortega, V.H. (1971, 26 de abril). El montaje III. Semanario Universidad, 2.

CINE; MONTAJE CINEMATOGRÁFICO- MODOS DE EXPRESIÓN.

BLDT $378 \mathrm{U}$

066

Acuña-Ortega, V.H. (1971, 10 de mayo,). Ingmar Bergman IV: 'Noche de circo'. Semanario Universidad, 2.

BERGMAN, INGMAR - OBRA; CINE SUECO

BLDT $378 \mathrm{U}$

067

Acuña-Ortega, V.H. (1971, 24 de mayo). Ingmar Bergman V: 'El sétimo sello'. Semanario Universidad, 2.

CRITICA DE CINE; BERGMAN, INGMAR; COMEDIA DRAMATICA; CINE SUECO

BLDT $378 \mathrm{U}$

068

Acuña-Ortega, V.H. (1971, 7 de junio,). Ingmar Bergman VII: Las fresas silvestres. Semanario Universidad,4.

CRITICA DE CINE; BERGMAN, INGMAR; COMEDIA DRAMATICA; CINE SUECO

BLDT

$378 \mathrm{U}$

069

Acuña-Ortega, V.H. (1971, 21 de junio). La batalla de Argel. Semanario Universidad, 4.

CRITICA DE CINE; PONTECORVO, GILLO

BLDT

$378 \mathrm{U}$

070

Acuña-Ortega, V.H. (1971, 12 de julio). Cine y época actual. Semanario Universidad, 4. 
CINE CONTEMPORÁNEO

BLDT

$378 \mathrm{U}$

071

Acuña-Ortega, V.H. (1971, 26 de julio). Luis Buñuel: La vía láctea. Semanario Universidad, 4.

BUÑUEL PORTOLÉS, LUIS; CRITICA DE CINE

BLDT

$378 \mathrm{U}$

072

Acuña-Ortega, V.H. (1971, 20 de setiembre). Periodismo e Historia. Semanario Universidad, 4.

GAMBOA, FRANCISCO; COMENTARIOS

BLDT

$378 \mathrm{U}$

073

Acuña-Ortega, V.H. (1971, 20 setiembre). Quilapayun: Un cantar revolucionario. Semanario Universidad, 5.

ESCUDERO, RUBEN - ENTREVISTA; CONJUNTO QUILAPAYUN

BLDT

$378 \mathrm{U}$

074

Acuña-Ortega, V.H. (1971, 1 de noviembre). Documentales canadienses. Semanario Universidad, 4.

CINE CANADIENSE; CORTO DOCUMENTAL; CORTO EXPERIMENTAL

BLDT

$378 \mathrm{U}$

075

Acuña-Ortega, V.H. (1971, 8 de noviembre). Historia tradicional e historia estructural. Semanario Universidad, 4.

COMENTARIOS; HALPERIN DOUGHI, TULIO

BLDT

$378 \mathrm{U}$ 
076

Acuña-Ortega, V.H.(1972, 24 de enero). Chacarita: una situación explosiva. Semanario Universidad, 5 .

CHACARITA, PUNTARENAS, COSTA RICA.

BLDT

$378 \mathrm{U}$

077

Acuña-Ortega, V.H. (1972, 17 de abril). Entrevista con el Decano de Derecho. Semanario Universidad, 5.

ORTIZ ORTIZ, EDUARDO - ENTREVISTA; CAMACHO; DANIEL - ENTREVISTA; ASUNTOS UNIVERSITARIOS; FACULTAD DE DERECHO, UNIVERSIDAD DE COSTA RICA

BLDT

$378 \mathrm{U}$

078

Acuña-Ortega, V.H. (1972, 24 de abril). Preeminencia de lo académico sobre lo administrativo. Semanario Universidad, 5.

ASUNTOS UNIVERSITARIOS; ACADEMIA

BLDT

$378 \mathrm{U}$

079

Acuña-Ortega, V.H. (1972, 8 de mayo,). Intelectuales colonizados. Semanario Universidad, 4.

INTELECTUALES LATINOAMERICANOS; ALINEACION IDEOLOGICA

BLDT

$378 \mathrm{U}$

080

Acuña-Ortega, V.H. (1972, 22 de mayo). Balance del tercer congreso. Semanario Universidad, 7.

CONGRESO UNIVERSITARIO. 3; UNIVERSIDAD DE COSTA RICA

BLDT

$378 \mathrm{U}$

081

Acuña-Ortega, V.H. (1989, 14 de julio). La Editorial y el futuro de la Universidad. Semanario Universidad, 5.

EDITORIAL DE LA UNIVERSIDAD DE COSTA RICA, EUCR 
$\mathrm{BN}$

082

Acuña-Ortega, V.H. (1992, 13 de marzo). Castigo divino, profano castigo. Semanario Universidad, 5.

CASTIGO DIVINO (LIBRO); COMENTARIOS; LITERATURA NICARAGÜENSE;RAMÍREZ MERCADO, SERGIO

$\mathrm{BN}$

083

Acuña-Ortega, V.H. (1993, 1 de diciembre). Pensar Centroamérica en una perspectiva histórica. Suplemento La Gaceta, 1

AMERICA CENTRAL; HISTORIA

$\mathrm{BN}$

084

Acuña-Ortega, V.H. (1993, 15 de diciembre). Pensar Centroamérica en una perspectiva histórica. Suplemento La Gaceta, 1.

AMERICA CENTRAL; HISTORIA

$\mathrm{BN}$

085

Acuña-Ortega, V.H. (1995, 8 de mayo). La intención de no callar. La Nación. Suplemento Viva, 11. MUJERES; FEMINISMO EN COSTA RICA (LIBRO); COMENTARIOS; BERRON, LINDA

BLDT

$0 \mathrm{~N}$

$\mathrm{BN}$

086

Acuña-Ortega, V.H. (1996, 10 de mayo). Final del juego (I). Semanario Universidad, 21

UNIVERSIDAD DE COSTA RICA - HISTORIA; CRISIS.

BLDT

$378 \mathrm{U}$ BN

087

Acuña-Ortega, V.H. (1996, 17 de mayo). Final del juego (II). Semanario Universidad, 18. 
UNIVERSIDAD DE COSTA RICA; CRISIS DE SOSTENIBILIDAD

BLDT

$378 \mathrm{U}$

BN

088

Acuña-Ortega, V.H. (1996, 24 de mayo). Final del juego (III). Semanario Universidad, 15.

UNIVERSIDAD DE COSTA RICA; GLOBALIZACION

BLDT

$378 \mathrm{U}$ BN

089

Acuña-Ortega, V.H. (1997, 2 de noviembre). Secularizacion política. La Nación. Suplemento Áncora, 5.

IDENTIDAD NACIONAL; SECULARIZACIÓN POLÍTICA; SOCIEDAD COSTARRICENSE; CIVISMO; IMPUNIDAD

BLDT

$0 \mathrm{~N}$.

BN

http://www.nacion.com/ancora/1997/noviembre/02/ancora5.html

090

Acuña-Ortega, V.H. (1998, 16 de setiembre). Uno y los otros de Álvaro Quesada. Semanario Universidad. Suplemento Los libros, 1-2.

QUESADA SOTO, ALVARO; UNO Y LOS OTROS, IDENTIDAD Y LITERATURA EN COSTA RICA, 1890-1940 (LIBRO); LITERATURA COSTARRICENSE; COMENTARIOS

BLDT

$378 \mathrm{U}$ BN

091

Acuña-Ortega, V.H. (1998, 30 de setiembre). Un día en la vida... de un profesor. Semanario Universidad, 14.

UNIVERSIDAD DE COSTA RICA - PROFESORADO

BLDT

$378 \mathrm{U}$

092

Acuña-Ortega, V.H. (1998, 29 de noviembre). Texto que provoca, interroga y convoca. La Nación. Suplemento Ancora, 3. 
PÉREZ-RATTON, VIRGINIA; COMENTARIOS

BLDT $0 \mathrm{~N}$

http://www.nacion.com/ancora/1998/noviembre/29/ancora3.html

093

Acuña-Ortega, V.H. (1999, 19 de Setiembre). Elogio del inmigrante. La Nación. Suplemento Áncora, 1-2.

INMIGRANTES; ENSAYOS COSTARRICENSES

BLDT $0 \mathrm{~N}$

BN

http://www.nacion.com/ancora/1999/septiembre/19/ancora1.html

094

Acuña-Ortega, V.H. (2000, 10 de mayo). Don Rafael. Semanario Universidad, 18.

OBREGON LORIA, RAFAEL; HISTORIADOR COSTARRICENSE

BLDT

$378 \mathrm{U}$

095

Acuña-Ortega, V.H. (2000, 08 de octubre). 20 años de Cantoamérica. La Nación. Suplemento Áncora, 4.

CANTOAMERICA; MONESTEL, MANUEL; MUSICA TROVA.

BLDT $0 \mathrm{~N}$

http://www.nacion.com/ancora/2000/octubre/08/ancora4.html

096

Acuña-Ortega, V.H. (2000, 26 de noviembre). El café en la cultura costarricense. La Nación. Suplemento Áncora, 1-3.

COSTA RICA - HISTORIA; CAFE - HISTORIA; IDENTIDAD COSTARRICENSE

BLDT $378 \mathrm{U}$

BN

http://www.nacion.com/ancora/2000/noviembre/26/ancora1.html

097

Acuña-Ortega, V.H. (2001, 7 de enero). El cuerpo del pasado. La Nación. Suplemento Áncora, 1-2. ARQUITECTURA; LA HISTORIA DE LA ARQUITECTURA EN COSTA RICA (LIBRO); COMENTARIOS 
$\mathrm{BN}$

http://www.nacion.com/ancora/2001/enero/07/ancora1.html

098

Acuña-Ortega, V.H. (2001, 18 de febrero). El arte de la ironía. La Nación. Suplemento Áncora, 4

QUESADA SOTO, ALVARO; CÍRCULO DE ESTUDIOS MARIO SANCHO

BLDT $0 \mathrm{~N}$

BN

http://www.nacion.com/ancora/2001/febrero/18/ancora7.html

099

Acuña-Ortega, V.H. (2001, 8 de abril). Mito de la nación costarricense. La Nación. Suplemento Áncora, 1-2.

COSTA RICA; IDENTIDAD NACIONAL; INVENCIÓN DE LA NACIÓN

BLDT

$0 \mathrm{~N}$

$\mathrm{BN}$

http://www.nacion.com/ancora/2001/abril/08/ancora2.html

100

Acuña-Ortega, V.H. (2001, 15 de julio). El álbum de Figueroa. La Nación. Suplemento Áncora, 1-2.

ALBUM DE FIGUEROA; FIGUEROA OREAMUNO, JOSE MARIA; COSTA RICA- SIGLO XIX; IMAGINARIO COSTARRICENSE.

BLDT

$0 \mathrm{~N}$

BN

http://www.nacion.com/ancora/2001/julio/15/ancora1.html

101

Acuña-Ortega, V.H. (2001, 19 de julio). Migraciones. La Nación, 14A.

EMIGRACION E INMIGRACION; NICARAGUENSES; COSTA RICA

$\mathrm{BN}$

http://www.nacion.com/ln_ee/2001/julio/19/opinion7.html

102

Acuña-Ortega, V.H. (2003, 23 de febrero). Vigencia de las Ciencias Sociales. La Nación. Suplemento Áncora, 4.

PROYECTO ESTADO DE LA NACION; INFORME; AGENDA NACIONAL; DESARROLLO SOSTENIBLE - COSTA RICA; PREMIO ANCORA 
BN

http://www.nacion.com/ancora/2003/febrero/23/ancora4.html

103

Acuña-Ortega, V.H. (2004, 15 de febrero). Rescate de identidad hacia el futuro. La Nación. Suplemento Áncora, 11 .

Nota: comentario sobre el libro Arquitectura rural en el trópico. Enclaves bananeros en Costa Rica.

STAGNO, BRUNO; UGARTE, JIMENA; ARQUITECTURA; COSTA RICA; ARQUITECTURA RURAL EN EL TROPICO; COMENTARIOS

BLDT $0 \mathrm{~N}$

http://www.nacion.com/ancora/2004/febrero/15/ancora9.html

104

Acuña-Ortega, V.H. (2004, 23 de noviembre). El futuro es posible. La Nación, 30 A.

FESTIVAL INTERNACIONAL DE LAS ARTES (COSTA RICA); FORO CENTROAMÉRICA: EL FUTURO ES POSIBLE.

BLDT $0 \mathrm{~N}$

http://www.nacion.com/ln_ee/2004/noviembre/23/opinion5.html

105

Acuña-Ortega, V.H. (2007, 16 de setiembre). Encuentros y desencuentro. La Nación. Suplemento Áncora.

ESGUEVA, ANTONIO; COMENTARIO LITERARIO; NICARAGUA; COSTA RICA; RÍO SAN JUAN; FRONTERAS; LITIGIO; HISTORIA

http://www.nacion.com/ancora/2007/septiembre/16/libros1237268.html

106

Acuña-Ortega, V.H. (2007, 26 de agosto). Relectura de 'Mamita Yunai'. La Nación. Suplemento Áncora, 4.

FALLAS, CARLOS LUIS, 1909-1966; MAMITA YUNAI; LITERATURA COSTARRICENSE

BLDT $0 \mathrm{~N}$

http://www.nacion.com/ancora/2007/agosto/26/estaedicion1214090.html 


\section{ARTíCULOS DE REVISTAS}

107

Acuña-Ortega, V.H. (1978). Historia económica del tabaco en Costa Rica: época colonial. Anuario de Estudios Centroamericanos. (4), 279-392.

PRODUCCION; COMERCIO; MONOPOLIOS; COSECHAS; VENTAS; TABACO; HISTORIA; PERIODO COLONIAL; ECONOMIA; ASPECTOS SOCIALES

BEFT

BLDT

Biblioteca Sede de Occidente

CIDCACS

CIHAC
$633.71 \mathrm{~A} 184 \mathrm{~h}$

$900 \mathrm{~A}$

Revista 900 A c. 1

04805.16

108

Acuña-Ortega, V.H. (1979).Aspectos institucionales del comercio colonial hispánico en el Siglo XVIII: mercantilismo y pacto colonial. Revista Estudios. 1(2), 53-61.

MERCANTILISMO - ESPANA; HISTORIA ECONOMICA; PERIODO COLONIAL

BLDT

$900 \mathrm{R}$

109

Acuña-Ortega, V.H. (1980). Capital comercial y comercio exterior en América Central durante el siglo XVIII: una contribución. Estudios Sociales Centroamericanos. 9(26), 71-102.

CAPITAL; COMERCIO EXTERIOR; COMERCIO INTERNACIONAL PUBLICO; COMERCIO INTERNACIONAL PRIVADO; DERECHO COMERCIAL; AMERICA CENTRAL; HISTORIA ECONOMICA; ACTIVIDADES MERCANTILES

Biblioteca de Derecho - UCR 300E

CIDCACS

110

Acuña-Ortega, V.H. (1980). Reglamentación del comercio exterior en América Central durante el siglo XVIII. Mesoamérica. 1(1), 7-55.

COMERCIO INTERNACIONAL; CONVENIOS COMERCIALES; PERIODO COLONIAL; ESPAÑA; GUATEMALA; HISTORIA ECONOMICA; COMERCIO EXTERIOR; COMERCIO MARITIMO

CIHAC 
FBEH

111

Acuña-Ortega, V.H. (1981). Vladimir de la Cruz y la interpretación socialdemócrata de nuestra historia. Revenar. 1(3), 10-11.

CRUZ DE LEMOS, VLADIMIR DE LA; LUCHA DE CLASES; CRÍTICA; MOVIMIENTOS SOCIALES

BEFT

112 Acuña-Ortega, V.H. (). "Rodrigo Facio: un historiador vigente”. Revenar. 1(4): 10-12, 1981.

FACIO BRENES, RODRIGO

BEFT

113

Acuña-Ortega, V.H.(1985). Clases sociales y conflicto social en la economía cafetalera costarricense: productores contra beneficiadores, 1932-36. Revista de Historia. (Especial), 181-206.

Nota: El número de esta revista corresponde al simposio Historia; problemas y perspectiva agraria en Costa Rica.

CLASES SOCIALES; CONFLICTOS SOCIALES; ECONOMIA; CAFE; IDEOLOGIAS; ESTADO

BEFT

BLDT $900 \mathrm{R}$

BN

H 905 R4547r C.R.

Biblioteca Sede de Occidente

REVISTA 900 R c.1

CIDCACS

CIHAC

114

Acuña-Ortega, V.H. (1985). En el 65 aniversario de las huelgas por la jornada de las ocho horas. Aportes. 5(25), 26-28.

HUELGAS; JORNADAS DE TRABAJO; MOVIMIENTO OBRERO; COSTA RICA

$\mathrm{BN}$

H 301 A644a C.R.

CIDCACS

115

Acuña-Ortega, V.H. (1985 - 1986). Renovación de los estudios históricos en Costa Rica. Revista de Historia, 6-7(12-13), 11-16.

HISTORIA; GENERACIONES; HISTORIADORES 
116

Acuña-Ortega, V.H. (1986). Patrones del conflicto social en la economía cafetalera costarricense (1900-1948). Revista de Ciencias Sociales. (31), 113-122.

PROBLEMAS SOCIALES; PAISES EN DESARROLLO; ESTRATEGIA DEL DESARROLLO; ESTADO, CONDICIONES DE VIDA; ASPECTOS SOCIALES; MOVIMIENTOS SOCIALES

BEFT

BLDT

$\mathrm{BN}$

Biblioteca Sede de Occidente

CIDCACS

CIHAC
CR-CIDCACS: 09827.08

$300 \mathrm{R}$

H 300.5 R454r C.R.

REVISTA 300 R c.1

09827.08

117

Acuña-Ortega, V.H. (1987). Cerdas Cruz, Rodolfo. La hoz y el machete, la Internacional Comunista, América Latina y la Revolución en Centroamérica. Revista de Historia. (15), 171-176.

COMUNISMO; LIBROS; ANALISIS DE CONTENIDO

BLDT

900 R CIHAC

118

Acuña-Ortega, V.H. (1987). Relaciones entre asentamientos precolombinos al norte de Guayabo de Turrialba en la Fase Cabaña. Revista de Ciencias Sociales. (35), 43-52.

ARQUEOLOGIA; CIENCIAS SOCIALES; VALORES CULTURALES; FACTORES CULTURALES; INVESTIGACION

CIHAC

119

Acuña-Ortega, V.H. (1987). La ideología de los pequeños y medianos productores cafetaleros costarricenses. 1900-1961. Revista de Historia. (16), 137-159.

HISTORIA; CAFE; IDEOLOGIAS; PRODUCCION

BEFT

BLDT

BN

c. 1

CIHAC

H 905 R4547r C.R.

REVISTAS 15 
120 Acuña-Ortega, V.H. (1987). La responsabilidad social de la investigación social. Revenar. $7(13), 45-50$.

\section{CIENCIAS SOCIALES - INVESTIGACIONES}

BN

H 860.5 R451r C.R.

121

Acuña-Ortega, V.H. (1988). Vida cotidiana, condiciones de trabajo y organización sindical: el caso de los zapateros en Costa Rica, 1934-1955. Revista de Historia. (Especial), 223-244.

INDUSTRIA DEL CALZADO; CUERO; CONDICIONES DE TRABAJO; SINDICATOS; HISTORIA

BEFT

BLDT

BN

CIDCACS

CIHAC

Revista 900R

H 905 R4547r C.R.

12418.05

REVISTAS 15

122

Acuña-Ortega, V.H. (1989). Fuentes orales e historia obrera: el caso de los zapateros en Costa Rica. Secuencia. (13), 163-173.

ANALISIS HISTORICO; HISTORIA SOCIAL; PROBLEMAS SOCIALES; CLASE OBRERA; SINDICALISMO

CIDCACS

10826.01-04

123

Acuña-Ortega, V.H. (1989). Democracia y cultura política en Costa Rica. Revista Nacional de Cultura. (5), 56-59.

DEMOCRACIA; CULTURA POLITICA; COSTA RICA

BEFT

$\mathrm{BN}$

H 056 R4545r C.R.

124

Acuña-Ortega, V.H. (1991). La huelga de los tútiles, 1887-1889. Un capítulo de nuestra historia social; de Oscar Aguilar Bulgarelli. Revista de Historia. (23), 247-250.

MOVIMIENTOS SOCIALES; ANALISIS DE CONTENIDO; LIBROS

CIHAC REVISTAS 35 
125

Acuña-Ortega, V.H. (1993). La Historia y el fin de los mitos de las Ciencias Sociales. Reflexiones. (8), 29-36.

CAMBIO SOCIAL; HISTORIA; METODOLOGIA; EPISTEMOLOGIA

BEFT

$\mathrm{BN}$

CIDCACS

H 300.5 R332r C.R.

CIHAC

12819.01-05

126

Acuña-Ortega, V.H. (1993). Vocación y compromiso de un zapatero en Costa Rica. Revista de Historia. (27), 173-176.

MORALES ALFARO, JUAN RAFAEL; ETICA; CALZADO; BIOGRAFIAS

BEFT

BJGM

H 900 R

CIHAC

REVISTAS 15

127

Acuña-Ortega, V.H. (1994). Los desafíos de la historia en Centroamérica. Reflexiones. (20), 3-16.

HISTORIA; HISTORIOGRAFIA; AMERICA CENTRAL

BEFT

$\mathrm{BN}$

H 300.5 R332r C.R.

CIDCACS

$12828.01-03$

CIHAC

128

Acuña-Ortega, V.H. (1994). Crecimiento económico y pobreza: Centroamérica, 1870-1945. Reflexiones. (25), 25-38.

CRECIMIENTO ECONOMICO; POBREZA; REFORMA; HISTORIA ECONOMICA

BEFT

$\mathrm{BN}$

CIDCACS

H 300.5 R332r C.R.

CIHAC

12833.01-04

129

Acuña-Ortega, V.H. (1995). Nicaragua: identidad y política. Actualidades del CIHAC. 1(10).

IDENTIDAD; HISTORIA 
Laboratorio de Etnología - UCR Revista Actualidad del CIHAC n.10

130

Acuña-Ortega, V.H. (1996). Arqueología del comunismo a la tica. Actualidades del CIHAC. 3(2).

COMUNISMO; HISTORIA POLITICA

CIHAC

131

Acuña-Ortega, V.H. (1996). La historia social en Costa Rica: balances y perspectivas. Revista de Historia. (Especial), 141-149.

HISTORIA SOCIAL; CONFLICTOS SOCIALES; HISTORIOGRAFIA; COSTA RICA

BEFT

BJGM

BLDT

Biblioteca Sede de Occidente

CIHAC
H $900 \mathrm{R}$

$900 \mathrm{R}$

REVISTA 900 R c.1

REVISTAS 15

132

Acuña-Ortega, V.H. (1996). Sandino, una biografía política, de Volker Wünderich. Revista de Historia. (33), 201-205.

WUNDERICH, VOLKER; BIOGRAFIA POLITICA; SANDINO, AUGUSTO CESAR, 18951934; COMENTARIOS; LIBROS

Biblioteca Sede de Occidente

REVISTA 900 R c.1

CIHAC

133

Acuña-Ortega, V.H. (1996). Universidad: dinero, poder, saber y futuro. Reflexiones. (47), 23-33.

UNIVERSIDADES; ESTADO; HISTORIA

BEFT

CIDCACS

12849.01-04

CIHAC

134

Acuña-Ortega, V.H. (1998). Malestares e identidades: uno y los otros, de Álvaro Quesada. Revista de Historia. (38), 199-203. 
COMENTARIOS; LIBROS; QUESADA SOTO, ALVARO

$\mathrm{BN}$

H 905 R4547r C.R.

CIHAC

135

Acuña-Ortega, V.H. (1999). Elogio del inmigrante. Revista de Historia. (40), 1724.

INMIGRANTES; CULTURA; HISTORIA; MIGRACION; CONFERENCIAS

BEFT

BJGM

BLDT

$\mathrm{BN}$

CIDCACS

CIHAC

136

Acuña-Ortega, V.H. (1999-2000). Elogio del inmigrante. Herencia. 11(2) - 12(1-2), 1116.

INMIGRANTES; CULTURA; HISTORIA; MIGRACION; CONFERENCIAS

BLDT

$\mathrm{BN}$

CIHAC

137

Acuña-Ortega, V.H. (2000). Las concepciones de la comunidad política en Centroamérica en tiempos de la Independencia (1820-1823). Trace. (37), 27-40.

HISTORIA POLITICA; INDEPENDENCIA; PERIODO COLONIAL; DESCOLONIZACION; CLASES SOCIALES

CIHAC

REVISTAS 25

138

Acuña-Ortega, V.H. (2001). El álbum de Figueroa, rebeldía y fantasía en la Costa Rica del siglo XIX. Revista del Archivo Nacional. 65(1-12), 147-150.

FIGUEROA OREAMUNO, JOSE MARIA; HISTORIA; SIGLO XIX; ALBUM DE FIGUEROA BEFT

BN

H 905 R4547rea C.R. 
139

Acuña-Ortega, V.H. (2001). Costa Rica en imágenes. Revista del Archivo Nacional. 65(1-12), 139145.

HISTORIA; SIGLO XIX; FOTOGRAFIA; ASPECTOS CULTURALES; IDENTIDAD CULTURAL; ALBUM DE FIGUEROA

BEFT

$\mathrm{BN}$

H 905 R4547rea C.R.

140

Acuña-Ortega, V.H. (2001). Ofelia Sanou. Arquitectura e historia en Costa Rica: templos parroquiales en el Valle Central. Grecia, San Ramón y Palmares (1860-1914). Revista de Historia. (44), 249-251.

SANOU ALFARO, OFELIA; ARQUITECTURA E HISTORIA EN COSTA RICA; COMENTARIOS; ARQUITECTURA RELIGIOSA

BLDT $900 \mathrm{R}$

BN H 905 R4547 C.R.

Biblioteca Sede de Occidente $\quad 900$ R c.1

141

Acuña-Ortega, V.H. (2001). Ofelia Sanou Alfaro. Arquitectura e historia en Costa Rica. Ingenieros y Arquitectos. 45(16): 30-31.

SANOU ALFARO, OFELIA; ARQUITECTURA E HISTORIA EN COSTA RICA; COMENTARIOS; ARQUITECTURA RELIGIOSA

$\mathrm{BN}$

H 620.6 I-46-i

142

Acuña-Ortega, V.H.; Díaz-Arias, D.G. (2002). Identidades nacionales en Centroamérica: bibliografía de los estudios historiográficos. Revista de Historia. (45), 267-283.

BIBLIOGRAFIAS; DOCUMENTOS; HISTORIOGRAFÍA; AMERICA CENTRAL

$\mathrm{BN}$

Biblioteca Sede de Occidente

CIDCACS

CIHAC
H 905 R4547r C.R.

REVISTA 900 R c.1

19242.01-07 c2

143

Acuña-Ortega, V.H. (2002). La invención de la diferencia costarricense, 18101870. Revista de Historia. (45), 191-228. 
$\mathrm{BN}$

Biblioteca Sede de Occidente

CIDCACS

CIDCSO

CIHAC
H 905 R4547r C.R.

REVISTA 900 R c.1

19242.01-07 c2

144

Acuña-Ortega, V.H. (2002). Historia y ciudadanía. Humanidades. (1), 42-50.

HISTORIA; IDENTIDAD CULTURAL; SOCIEDAD; FORMACION PROFESIONAL

CIHAC

145

Acuña-Ortega, V.H. (2004). Lara Putnam: Company they kept. Migrants and the politics of gender in Caribbean Costa Rica, 1870-1960. Estudios Interdisciplinarios de América Latina y el Caribe. 15(2), 199-202.

BIBLIOGRAFIA; CITAS; PUTNAM, LARA; COMENTARIOS; MIGRANTES

CIHAC

146

Acuña-Ortega, V.H. (2004). Tiempo histórico y ciencias sociales en Centroamérica en la segunda mitad del siglo XX. Revista Centroamericana de Ciencias Sociales. 1(1), 9-24.

HISTORIA; CIENCIAS SOCIALES, CAMBIO SOCIAL; AMERICA CENTRAL; ANALISIS HISTORICO; COMENTARIOS; TEORIA SOCIAL

BEFT

BJGM

H 300.5 R454re

$\mathrm{BN}$

H 302.072 R454r C.R.

CIDCACS

REV1627

147

Acuña-Ortega, V.H. (2006). Memorias comparadas: las versiones de la guerra contra los filibusteros en Nicaragua, Costa Rica y Estados Unidos (Siglos XIX-XXI). Revista de Historia [Nicaragua]. (20-21), 5-21.

HISTORIA; FILIBUSTEROS; NICARAGUA; COSTA RICA; ESTADOS UNIDOS;GUERRA, HISTORIOGRAFIA; MEMORIA NACIONAL; CAMPAÑA NACIONAL; WALKER, WILLIAM

CIDCACS

REV1052 (20-21)

CIDCSO 


\section{REUNIONES, CONGRESOS, CONFERENCIAS, SEMINARIOS Y PONENCIAS}

148

Acuña-Ortega, V.H. (1986). La responsabilidad social de la investigación social. En: I Seminario sobre la investigación. San José, C.R.: Universidad de Costa Rica, Facultad de Ciencias Sociales.

Nota: material mimeografiado.

CIENCIAS SOCIALES - INVESTIGACIONES

CIDCSO 003256

149

Acuña-Ortega, V.H. (1988). Democracia y cultura política en Costa Rica. En: Simposio sobre Democracia y cultura política en Costa Rica. San José, C.R.: Ministerio de Cultura, Juventud y Deportes. (155-162).

DEMOCRACIA; CULTURA; POLITICA; SISTEMAS POLITICOS; SOCIEDAD; IDEOLOGIAS POLITICAS; HISTORIA; SISTEMAS ELECTORALES

CIHAC

04.02.02 3337 SIMPOSIOS

150

Acuña-Ortega, V.H. (1996). Nación y política en el comunismo costarricense (1930-1948). En: Congreso Centroamericano de Historia, III, Mesa \#3. San José, C.R.: UCR.

Nota: Mesa \#3, Historia de los movimientos sociales.

POLITICA; COMUNISMO; MOVIMIENTOS SOCIALES; HISTORIA; CONGRESO CENTROAMERICANO DE HISTORIA

CIHAC 05.01.01 1061 CONGRESOS-MESA no. 3

151

Acuña-Ortega, V.H. (2005). El Salvador y Costa Rica en la historiografía de Lorenzo Montúfar: construcción del estado e invención de la nación. En: Memoria del Primer Encuentro de Historia de El Salvador. San Salvador: CONCULTURA. (111-117).

HISTORIA; ANALISIS HISTORICO; BIBLIOGRAFIAS; MONTUFAR, LORENZO; IDENTIDAD CULTURAL; SISTEMAS POLITICOS; PRENSA; POLITICO GUATEMALTECO; EDUCADOR GUATEMALTECO

CIHAC 05.01.01 2859 ENCUENTROS 
152

Acuña-Ortega, V.H.; Martínez-Merino, J. (Eds.). (2005). Universidad de Costa Rica. Sede Guanacaste. Memoria. Primer encuentro de historia local de Guanacaste. [¿San José, C.R.?]: UCR.

HISTORIA; POLITICA; SOCIEDAD; INSTITUCIONES PÚBLICAS; IDENTIDAD;CULTURA; ENSEÑANZA SECUNDARIA; PROVINCIA DE GUANACASTE

BEFT

01.04 .0317499

153

Acuña-Ortega, V.H. (2007). Walker en Centroamérica según la historiografía filibustera. En:

Memoria. Simposio Internacional. Filibusterismo y destino manifiesto en las Américas.

Liberia, Guanacaste. (163-179).

Nota: material inédito.

HISTORIA; CAMPAÑA NACIONAL; FILIBUSTEROS; HISTORIOGRAFIA; WALKER, WILLIAM; AMERICA CENTRAL

MHJS

154

Acuña-Ortega, V.H. (Ed.). (2007). Memoria. Simposio internacional. Filibusterismo y destino manifiesto en las Américas. Liberia, Guanacaste.

Nota: material inédito localizado en el Museo Histórico Juan Santamaría

HISTORIA; CAMPAÑA NACIONAL 1856-1857; FILIBUSTEROS

MHJS

155

Acuña-Ortega, V.H. (2008). Memorias comparadas: las versiones de la guerra contra los filibusteros en Nicaragua, Costa Rica y Estados Unidos (Siglos XIX-XXI). En prensa.

Nota: Conferencia sobre Memoria e Identidad impartida en El Farolito, Centro Cultural Español, el 11 de noviembre del 2008.

MEMORIA HISTORICA; FILIBUSTEROS; NICARAGUA; COSTA RICA; ESTADOS UNIDOS; SIGLO XIX; SIGLO XX; SIGLO XXI

Colección del autor 


\section{DOCUMENTOS NO CONVENCIONALES}

156

Acuña-Ortega, V.H. (1980). La acumulación originaria y el desarrollo del capitalismo [grabación]. San Ramón, C.R.: UCR.

Nota: casete

CAPITALISMO; ECONOMIA; HISTORIA; GRABACION SONORA

Biblioteca sede de Occidente

330.122 A184a

157

Acuña-Ortega, V.H. [1985]. Historia del vocabulario político en Costa Rica [grabación]. San Ramón, C.R.: UCR.

Nota: casete

CIENCIAS POLITICAS; GLOSARIOS, VOCABULARIOS, ETC.; COSTA RICA; POLITICA Y GOBIERNO; GRABACION SONORA

Biblioteca Sede de Occidente 320.03 a184H No. 1

158

Programa 5 de febrero 2006 [grabación]. San José, C.R.: UCR : Radio Universidad de Costa Rica: Radio 101.9 en tu mundo

Nota: Parte 2 Entrevistas Mercedes Muñoz y Víctor Hugo Acuña Ortega. Disco compacto

POLITICOS COSTARRICENSES - DISCOS COMPACTOS; CIUDADANIA - COSTA RICA

- DISCOS COMPACTOS; PROCESO ELECTORAL - DISCOSCOMPACTOS; GRABACION SONORA; ACUÑA ORTEGA, VICTOR HUGO; MUÑOZ GUILLEN, MERCEDES

BNAU-CD

324 P9641-p C.R.

\section{DOCUMENTOS RECUPERADOS EN INTERNET}

159

Acuña-Ortega, V.H. (1966). Centroamérica: raíces autoritarias y brotes democroticos [sic]. Revista ENVIOS, [sic]. Recuperado de

http://www.archivochile.com/America_latina/al_vg/america_latina_dg_00030.pdf [Consulta 20 jun 2008]

AMERICA CENTRAL; DEMOCRACIA 
160

Acuña-Ortega, V.H. (1982). Capital comercial y comercio exterior en Centroamérica durante el siglo XVIII. Mesoamérica. (4), 302-331. Recuperado de http://www.mesoamericarevista. org/publicacion4.htm\#VictorAcunaOrtega [Consulta 9 nov 2008]

Nota: el resumen se recupera en formato html.

CAPITAL; COMERCIO EXTERIOR; COMERCIO INTERNACIONAL PUBLICO; COMERCIO INTERNACIONAL PRIVADO; DERECHO COMERCIAL; AMERICA CENTRAL; HISTORIA ECONOMICA; ACTIVIDADES MERCANTILES

161

Acuña-Ortega, V.H. (1989). Fuentes orales e historia obrera. El caso de los zapateros en Costa Rica. Secuencia. (13), 163-173. Recuperado de

http://www.institutomora.edu.mx/secuencia/pdf/13/13_09.pdf [Consulta 20 jun 2008]

HISTORIA SOCIAL; ZAPATEROS; COSTA RICA; CLASE OBRERA; ARTESANOS

162

Acuña-Ortega, V.H. [2004?]. La historiografía liberal centroamericana: la obra de Lorenzo Montúfar (1823-1898). Istmo. (12). Recuperado de

http://collaborations.denison.edu/istmo/n12/articulos/historiografia.html\#end00 [Consulta 20 jun 2008]

\section{HISTORIOGRAFIA; MONTUFAR, LORENZO}

163

Acuña-Ortega, V.H. (2004). Tiempo histórico y ciencias sociales en Centroamérica en la segunda mitad del siglo XX: Encuentros. Revista Centroamericana de Ciencias Sociales. 1(1), 9-24. Recuperado de http://www.flacso.or.cr/fileadmin/documentos/FLACSO/Revista_CSS-1. pdf [Consulta 6 set 2008]

HISTORIA; AMÉRICA CENTRAL; REVISISON DE LITERATURA; SIGLO XX; CIENCIAS SOCIALES

164

Acuña-Ortega, V.H. (2005). Las concepciones de la comunidad política en Centroamérica en tiempos de la independencia (1820-23). Por F. Colom-González. Relatos de nación: la construcción de las identidades nacionales en el mundo hispánico (Tomo 1). Madrid / Frankfurt: Iberoamericana. (251-273). Recuperado de http://www.ibero-americana.net/ files/pdf/521222.pdf [Consulta 9 nov 2008]

Nota: el índice se recupera en formato pdf 
HISTORIA POLITICA; INDEPENDENCIA; PERIODO COLONIAL; DESCOLONIZACION; CLASES SOCIALES

165

Acuña-Ortega, V.H. (2005). Vertientes del recuerdo: Nicaragua, Walker y Costa Rica (siglos XIXXXI). En: Programa Estado, Nación e Identidades en América Central. Universidad de Costa Rica, CIHAC, 2005. Recuperado de

http://64.233.179.104/scholar?hl=es\&lr=\&q=cache:lqs3pUSz6QJ:www.historia.fcs.ucr. ac.cr/hcostarica/2005/Vertienconf.doc [Consulta 20 jun 2008]

CAMPAÑA NACIONAL; COSTA RICA; NICARAGUA; WALKER, WILLIAM; REVISION DE LITERATURA

166

Acuña-Ortega, V.H. (2006). Un empate insólito. Infolatam. Recuperado de http://www.infolatam. com/entrada/un_empate_insolito-246.html [Consulta 20 jun 2008]

COSTA RICA; CAMPAÑA POLITICA; ELECCIONES PRESIDENCIALES PONGAEL NOMBRE DEL CANDIDATO Ó CANDIDATOS

167

Acuña-Ortega, V.H. (2006). La historiografía liberal centroamericana: la obra de Lorenzo Montúfar (1823-1898). Historia y Sociedad (Medellín). (12). Recuperado de http://nemesis.unalmed. edu.co/publicaciones/revistas/historiaysociedad/ediciones/articulos_revista_1 2/victor_ hugo_acuna.pdf [Consulta 9 nov 2008]

Nota: pronto se encontrará disponible en la BLDT, en este momento se encuentra en el Departamento de Catalogación

LIBERALISMO; HISTORIOGRAFIA; REGIMEN DE HISTORICIDAD; AMÉRICA CENTRAL

168

Acuña-Ortega, V.H. (2006). Vertientes del recuerdo: historia y memoria de la guerra contra los filibusteros: Estados Unidos, Nicaragua y Costa Rica (siglos XIX-XXI). Recuperado de http://cihac.fcs.ucr.ac.cr/documentos/ppacifico/vertientes.pdf [Consulta 22 oct 2008]

Nota: versión preliminar

HISTORIOGRAFÍA; COSTA RICA; NICARAGUA; ESTADOS UNIDOS; WALKER, WILLIAM

169

Acuña-Ortega, V.H. (2006). Costa Rica: jal fin hay presidente!. Infolatam. Recuperado de http:// www.infolatam.com/entrada/costa_rica_al_fin_hay_presidente-396.html [Consulta 20 jun 2008] 
170

Acuña-Ortega, V.H. (2007). Historia y memoria de la guerra contra los filibusteros (siglos XIX-XXI). Boletín de la Asociación para el Fomento de los Estudios Históricos en Centroamérica (AFEHC). (31). Recuperado de http://afehc-historia-centroamericana.org/index.php?action=fi_aff\&id=1570 [Consulta 20 jun 2008]

FILIBUSTEROS; CAMPAÑA NACIONAL

171

Acuña-Ortega, V.H. (2007). Mamita Yunai: Un cuarto de siglo después. Recuperado de http://cihac. fcs.ucr.ac.cr/documentos/ppacifico/mamitayunai.pdf También en: http://www.nacion. com/ancora/2007/agosto/26/estaedicion1214090.html [Consulta 20 jun 2008]

Nota: Conferencia pronunciada en el ciclo Tertulias Literarias del Farolito. Lecturas y Relecturas de la Literatura Costarricense. Las ficciones de la "no-ficción” el día jueves 26 de julio de 2007.

FALLAS, CARLOS LUIS; MAMITA YUNAI; COMENTARIO LITERARIO; CONFERENCIAS.

\section{COMENTARIOS SOBRE LA OBRA DEL AUTOR}

172

Flores-Macal, M. (1975, 5 de diciembre). El tabaco en Costa Rica. Excelsior. [¿p.?]

ACUÑA-ORTEGA, V.H.;COMENTARIOS; TESIS

BLDT

$378 \mathrm{U}$

173

Samper-Kutschbach, M. (1985). Enfrentamiento y conciliación: comentarios a propósito de las relaciones entre productores y beneficiadores de café. Revista de Historia. (Especial), 207212.

Nota: El número de esta revista corresponde al simposio Historia; problemas y perspectiva agraria en costa Rica.

ACUÑA-ORTEGA, V.H.;COSTA RICA; CAFICULTURA; CLASES SOCIALES; LUCHAS SOCIALES; PRODUCTORES DE CAFE; COMENTARIOS

$\mathrm{BN}$

H 905 R4547r C.R. 
174

Gaínza, G. (1988). Reproducción social, discursos e ideología. Revista de Historia . (17), 133-144.

CAFETALEROS; GONZALEZ ORTEGA, ALFONSO; ACUÑA ORTEGA, VÍCTOR HUGO

BN

H 905 R4547r C.R.

175

González-García, Y. (1988). Hacia un diálogo académico sobre el análisis de un discurso. Revista de Historia. (17), 145-153.

CAFETALEROS; GONZALEZ ORTEGA, ALFONSO; ACUÑA ORTEGA, VÍCTOR HUGO $\mathrm{BN}$

H 905 R4547r C.R.

176

Rojas-González, M. (1988). Texto y realidad: el análisis de textos en las ciencias sociales. Revista de Historia. (17), 155-159.

CAFETALEROS; GONZALEZ ORTEGA, ALFONSO; ACUÑA ORTEGA, VÍCTOR HUGO $\mathrm{BN}$ H 905 R4547r C.R.

177

Viales, R. (1993). La historia y el presente: a propósito de la historia y el fin de los mitos de las ciencias sociales de Víctor Hugo Acuña. Reflexiones. (8) 3743.

ANALISIS DE CONTENIDO; ACUÑA ORTEGA, VICTOR HUGO; CAMBIO SOCIAL;

HISTORIA; METODOLOGIA; EPISTEMOLOGIA

BEFT

$\mathrm{BN}$

CIDCACS

H 300.5 R332r C.R.

CIHAC

12819.01-05

178

Molina-Jiménez, I. (2002). Acuña Ortega, Víctor Hugo: Comunidad política e identidad política en Costa Rica en el siglo XIX. Biblio 3W, Revista Bibliográfica de Geografía y Ciencias Sociales, 7(367). Recuperado de http://www.ub.es/geocrit/b3w-367.htm [Consulta 20 jun 2008]

IDENTIDAD NACIONAL; POLÍTICA; COSTA RICA; ACUÑA ORTEGA, VICTOR HUGO 


\section{BIBLIOGRAFÍA}

Acuña, V. H. (2007). Curriculum Vitae. Costa Rica.

Acuña, V. H. (04 de agosto de 2008). Entrevista sobre la Vida del Autor. (V. A. Barrientos, Entrevistador) Costa Rica.

Arquero, R. (2005). Biobibliografía del profesor Dr. José Luis del Río Sadornil. Disponible en http://revistas.ucm.es/inf/02104210/articulos/DCIN0505110017A.PDF

Delgado, J. (2005). Introducción a la biblografía. Los repositorios bibliográficos y su elaboración. España: Arco/libros.

Díez, I. (2000). Las bio-bibliografías: estado actual y metodología. Cuadernos de documentación Multimedia, 1(1), 67-77.

González, R. (2013). Guía práctica para elaborar ctas y referencias bibliográficas: adaptado al estilo APA. Costa Rica: Editorial Universidad de Costa Rica.

Pineda, S. y Roldán, B. (1994). Guía de investigación documental. Costa Rica:EUCR [Material mimeografiado].

Unfried, A. (2006). Biobibliografía de la escritora costarricense Virginia Grütter. Costa Rica: EUCR.

\section{-SOBRE LA AUTORA:}

Vanessa Alvarado-Barrientos es Bachiller en Bibliotecología por la Universidad de Costa Rica. Correo electrónico: vaneab.tica@gmail.com 